\title{
Minimal crystallizations of 3-manifolds
}

\author{
Biplab Basak Basudeb Datta \\ Department of Mathematics \\ Indian Institute of Science \\ Bangalore 560 012, India \\ biplab10@math.iisc.ernet.in \\ dattab@math.iisc.ernet.in
}

Submitted: Dec 10, 2013; Accepted: Mar 1, 2014; Published: Mar 17, 2014

Mathematics Subject Classifications: 57Q15; 57Q05; 57N10; 05C15

\begin{abstract}
We have introduced the weight of a group which has a presentation with number of relations is at most the number of generators. We have shown that the number of facets of any contracted pseudotriangulation of a connected closed 3-manifold $M$ is at least the weight of the fundamental group of $M$. This lower bound is sharp for the 3-manifolds $\mathbb{R P}^{3}, L(3,1), L(5,2), S^{1} \times S^{1} \times S^{1}, S^{2} \times S^{1}, S^{2} \times S^{1}$ and $S^{3} / Q_{8}$, where $Q_{8}$ is the quaternion group. Moreover, there is a unique such facet minimal pseudotriangulation in each of these seven cases. We have also constructed contracted pseudotriangulations of $L(k q-1, q)$ with $4(q+k-1)$ facets for $q \geqslant 3$, $k \geqslant 2$ and $L(k q+1, q)$ with $4(q+k)$ facets for $q \geqslant 4, k \geqslant 1$. By a recent result of Swartz, our pseudotriangulations of $L(k q+1, q)$ are facet minimal when $k q+1$ are even. In 1979, Gagliardi found presentations of the fundamental group of a manifold $M$ in terms of a contracted pseudotriangulation of $M$. Our construction is the converse of this, namely, given a presentation of the fundamental group of a 3-manifold $M$, we construct a contracted pseudotriangulation of $M$. So, our construction of a contracted pseudotriangulation of a 3 -manifold $M$ is based on a presentation of the fundamental group of $M$ and it is computer-free.
\end{abstract}

Keywords: Pseudotriangulations of manifolds, Crystallizations, Lens spaces, Presentations of groups.

\section{Introduction and Results}

A simplicial cell complex $K$ of dimension $d$ is a poset isomorphic to the face poset $\mathcal{X}$ of a $d$-dimensional simplicial CW-complex $X$. The topological space $X$ is called the geometric carrier of $K$ and is also denoted by $|K|$. If a topological space $M$ is homeomorphic to $|K|$, then $K$ is said to be a pseudotriangulation of $M$. For $d \geqslant 1$, a $(d+1)$-colored contracted graph $\Gamma=(V, E)$ with an edge coloring $\gamma: E \rightarrow\{1, \ldots, d+1\}$ determines a 
$d$-dimensional simplicial cell complex $\mathcal{K}(\Gamma)$ whose vertices have one to one correspondence with the colors $1, \ldots, d+1$ and the facets have one to one correspondence with the vertices in $V$. If $\mathcal{K}(\Gamma)$ is a pseudotriangulation of a space $M$ then $(\Gamma, \gamma)$ is called a crystallization of $M$. So, if $(\Gamma, \gamma)$ is a crystallization of a $d$-manifold $M$ then the number of vertices in the pseudotriangulation $\mathcal{K}(\Gamma)$ of $M$ is $d+1$. In [15], Pezzana showed the following.

Proposition 1 (Pezzana). Every connected closed PL-manifold admits a crystallization.

Thus, every connected closed $\mathrm{pl} d$-manifold has a contracted pseudotriangulation, i.e., a pseudotriangulation with $d+1$ vertices. In this article, we are interested in crystallizations of connected closed 3-manifolds with minimum number of vertices.

In [6], Epstein proved that the fundamental group of a 3-manifold has a presentation with the number of relations less than or equal to the number of generators. For such a group $G$, we define the weight $\psi(G)$ of $G$ in Definition 10 below. The weight of the trivial group is 2 and $\psi(G) \geqslant 8$ for any non-trivial group $G$ as we see later.

Definition 2. For a connected closed 3-manifold $M$, let $\psi(M)$ be the weight $\psi(\pi(M, x))$ of the group $\pi(M, x)$ for some $x$ in $M$.

If $M$ and $N$ are homeomorphic then clearly $\psi(M)=\psi(N)$. Thus, $\psi(M)$ is a topological invariant. Clearly, $\psi\left(S^{3}\right)=2$ and, in view of Perelman's theorem (Poincaré conjecture) [14], $\psi(M) \geqslant 8$ for $M \neq S^{3}$. Here, we have the following.

Lemma 3. Let $\psi(M)$ be as above and let $Q_{8}$ be the quaternion group $\{ \pm 1, \pm i, \pm j, \pm k\}$. Then $\psi\left(\mathbb{R P}^{3}\right)=\psi\left(S^{2} \times S^{1}\right)=\psi\left(S^{2} \times S^{1}\right)=8, \psi(L(3,1))=12, \psi(L(5, q))=16, \psi\left(S^{3} / Q_{8}\right)$ $=18, \psi\left(S^{1} \times S^{1} \times S^{1}\right)=24$ for $1 \leqslant q \leqslant 2$.

For a $d$-dimensional simplicial cell complex $K$, let $f_{j}(K)$ denote the number of $j$ cells of $K$ for $0 \leqslant j \leqslant d$. Let $g_{2}(K):=f_{1}(K)-(d+1) f_{0}(K)+\left(\begin{array}{c}d+2 \\ 2\end{array}\right)$ and $h_{2}(K):=$ $f_{1}(K)-d f_{0}(K)+\left(\begin{array}{c}d+1 \\ 2\end{array}\right)$. For a connected simplicial cell complex $K$, let $m(K)$ be the minimal number of generators of $\pi(|K|, *)$. For a connected closed pl $d$-manifold $M$, let

$$
\begin{aligned}
\Psi(M) & =\min \{m: M \text { has a crystallization with } m \text { vertices }\} \\
& =\min \left\{f_{d}(K): K \text { is a contracted pseudotriangulation of } M\right\} .
\end{aligned}
$$

In [11], Klee proved that $h_{2}(K) \geqslant\left(\begin{array}{c}d+1 \\ 2\end{array}\right) m(K)$ for any $d$-dimensional normal pseudomanifold $K$ whose edge graph is $(d+1)$-colorable. Here we have the following.

Theorem 4. Let $M$ be a connected closed 3-manifold. If $(\Gamma, \gamma)$ is a crystallization of $M$ then $\Gamma$ has at least $\psi(M)$ vertices. Equivalently, if $X$ is a contracted pseudotriangulation of $M$ then $f_{3}(X) \geqslant \psi(M)$.

Corollary 5. Let $M$ be a connected closed 3-manifold $M$ and $\mathbb{F}$ be a field. If $X$ is a contracted pseudotriangulation of $M$ then $g_{2}(X)=h_{2}(X) \geqslant \Psi(M)-2 \geqslant \psi(M)-2 \geqslant$ $6 m(M) \geqslant 6 \beta_{1}(M ; \mathbb{F})$. 
Consider the contracted pseudotriangulation $K_{1}:=\mathcal{K}\left(\mathcal{J}_{1}\right)$ of $S^{2} \times S^{1}$ corresponding to the crystallization $\mathcal{J}_{1}$ in Fig. 2 below. Since $f_{3}\left(K_{1}\right)=8$, it follows that $f_{2}\left(K_{1}\right)=16$ and hence $f_{1}\left(K_{1}\right)=12$. Therefore, $g_{2}\left(K_{1}\right)=12-16+10=6=6 \beta_{1}\left(S^{2} \times S^{1} ; \mathbb{Q}\right)$. Thus, the inequalities in Corollary 5 are equalities and (hence) the lower bound is sharp.

From the complete enumeration (obtained by using high-powered computers) of crystallizations of prime 3-manifolds with at most 30 vertices, we know $\Psi(M)$ for all closed prime 3-manifolds $M$ with $\Psi(M) \leqslant 30$ (cf. [3, 12]). In particular, we know that the minimal crystallizations of several 3-manifolds are unique and there are 3-manifolds which have more than one minimal crystallizations (see Remark 25 below). We have proved the existence and the uniqueness of some crystallizations using presentations of the fundamental groups. Consider a group $G$ which has a presentation with number of relations is at most the number of generators. From Theorem 4 we know that the number of vertices in any crystallization $(\Gamma, \gamma)$ of a closed connected 3-manifold $M$, whose fundamental group is $G$, is at least $\psi(G)$. We have constructed crystallizations on $\psi(G)$ vertices which yield presentations of $G$ as mentioned at the end of Section 2.4. We have considered the groups $\mathbb{Z}, \mathbb{Z}_{2}, \mathbb{Z}_{3}, \mathbb{Z}_{5}, \mathbb{Z}^{3}$ and $Q_{8}$ and have obtained such crystallizations. Generalizing some of these constructions, we have constructed two infinite families of crystallizations of lens spaces. More explicitly, we have the following.

Theorem 6. (i) If $M=\mathbb{R P}^{3}, S^{2} \times S^{1}, S^{2} \times S^{1}, L(3,1), L(5,2), S^{3} / Q_{8}$ or $S^{1} \times S^{1} \times S^{1}$ then $\Psi(M)=\psi(M)$ and $M$ has a unique contracted pseudotriangulation with $\psi(M)$ facets.

(ii) Let $X$ be a contracted pseudotriangulation of a connected closed 3-manifold $M$. If $f_{3}(X) \leqslant 8$ then $M$ is (homeomorphic to) $S^{3}, \mathbb{R P}^{3}, S^{2} \times S^{1}$ or $S^{2} \times S^{1}$.

Corollary 7. Let $X$ be a contracted pseudotriangulation of a closed 3-manifold $M$. If $M$ is $S^{3} / Q_{8}, S^{1} \times S^{1} \times S^{1}$ or $L(p, q)$ for some $p \geqslant 3$ then $h_{2}(X)>6 m(M)$.

Theorem 8. (i) $\Psi(L(k q-1, q)) \leqslant 4(k+q-1)$ for $k, q \geqslant 2$ and

(ii) $\Psi(L(k q+1, q)) \leqslant 4(k+q)$ for $k, q \geqslant 1$.

Remark 9. Recently, Swartz proved that $\Psi(L(k q+1, q)) \geqslant 4(k+q)$ whenever $k, q$ are odd $([16])$. Thus, $\Psi(L(k q+1, q))=4(k+q)$ for odd positive integers $k, q$. We found that $\Psi(L(5,1))=20=\Psi(L(7,2))$. So, Swartz's bound is also valid for $L(5,1)$ and $L(7,2)$. We also found that $\psi\left(\mathbb{Z}_{4}\right)=14$ and $\psi\left(\mathbb{Z}_{6}\right)=\psi\left(\mathbb{Z}_{7}\right)=18$. Proofs of these are in earlier versions of this article in the arXiv (arXiv:1308.6137). We have omitted these proofs from this version for the sake of brevity.

\section{Preliminaries}

\subsection{Colored Graphs}

All graphs considered here are finite multigraphs without loops. If $\Gamma=(V, E)$ is a graph and $U \subseteq V$ then the induced subgraph $\Gamma[U]$ is the subgraph of $\Gamma$ whose vertex set is $U$ and 
edges are those edges of $\Gamma$ whose end points are in $U$. For $n \geqslant 2$, an $n$-cycle is a closed path with $n$ distinct vertices and $n$ edges. If vertices $a_{i}$ and $a_{i+1}$ are adjacent in an $n$-cycle for $1 \leqslant i \leqslant n$ (addition is modulo $n$ ) then the $n$-cycle is denoted by $C_{n}\left(a_{1}, a_{2}, \ldots, a_{n}\right)$. A graph $\Gamma$ is called $h$-regular if the number of edges adjacent to each vertex is $h$.

An edge coloring of a graph $\Gamma=(V, E)$ is a map $\gamma: E \rightarrow C$ such that $\gamma(e) \neq \gamma(f)$ whenever $e$ and $f$ are adjacent (i.e., $e$ and $f$ are adjacent to a common vertex). The elements of the set $C$ are called the colors. If $C$ has $h$ elements then $(\Gamma, \gamma)$ is said to be an $h$-colored graph.

Let $(\Gamma, \gamma)$ be an $h$-colored graph with color set $C$. If $B \subseteq C$ with $k$ elements then the graph $\left(V(\Gamma), \gamma^{-1}(B)\right)$ is a $k$-colored graph with coloring $\left.\gamma\right|_{\gamma^{-1}(B)}$. This colored graph is denoted by $\Gamma_{B}$. Let $(\Gamma, \gamma)$ be an $h$-colored connected graph with color set $C$. If $\Gamma_{C \backslash\{c\}}$ is connected for all $c \in C$ then $(\Gamma, \gamma)$ is called contracted.

Let $\Gamma_{1}=\left(V_{1}, E_{1}\right)$ and $\Gamma_{2}=\left(V_{2}, E_{2}\right)$ be two disjoint $h$-regular $h$-colored graphs with same color set $\{1, \ldots, h\}$. For $1 \leqslant i \leqslant 2$, let $v_{i} \in V_{i}$. Consider the graph $\Gamma$ which is obtained from $\left(\Gamma_{1} \backslash\left\{v_{1}\right\}\right) \sqcup\left(\Gamma_{2} \backslash\left\{v_{2}\right\}\right)$ by adding $h$ new edges $e_{1}, \ldots, e_{h}$ with colors $1, \ldots, h$ respectively such that the end points of $e_{j}$ are $u_{j, 1}$ and $u_{j, 2}$, where $v_{i}$ and $u_{j, i}$ are joined in $\Gamma_{i}$ with an edge of color $j$ for $1 \leqslant j \leqslant h, 1 \leqslant i \leqslant 2$. (Here $\Gamma_{i} \backslash\left\{v_{i}\right\}=\Gamma_{i}\left[V_{i} \backslash\left\{v_{i}\right\}\right]$.) The colored graph $\Gamma$ is called the connected sum of $\Gamma_{1}, \Gamma_{2}$ and is denoted by $\Gamma_{1} \# v_{v_{1} v_{2}} \Gamma_{2}$.

Let $\Gamma=(V, E)$ be a $(d+1)$-regular graph with a $(d+1)$-coloring $\gamma: E \rightarrow C$. Let $x, y \in V$ be joined by $k$ edges $e_{1}, \ldots, e_{k}$, where $1 \leqslant k \leqslant d$. Let $B=C \backslash \gamma\left(\left\{e_{1}, \ldots, e_{k}\right\}\right)$. Let $X$ (resp., $Y$ ) be the components of $\Gamma_{B}$ containing $x$ (resp., $y$ ). If $X \neq Y$ then $\Gamma[\{x, y\}]$ is called a $d$-dimensional dipole of type $k$. Dipoles of types 1 and $d$ are called degenerate dipoles.

Let $\Gamma=(V, E)$ be a $(d+1)$-regular graph with a $(d+1)$-coloring $\gamma: E \rightarrow C$ and a dipole $\Gamma[\{x, y\}]$ of type $k$. Let $B, X$ and $Y$ be as above. A $(d+1)$-regular graph $\left(\Gamma^{\prime}, \gamma^{\prime}\right)$ with same color set $C$ is said to obtained from $\Gamma$ by cancelling the dipole $\Gamma[\{x, y\}]$ if (i) $\Gamma_{B}^{\prime}$ is obtained from $\Gamma_{B}$ by replacing $X \sqcup Y$ by $X \#_{x y} Y$, and (ii) two vertices $u, v$ of $\Gamma^{\prime}$ are joined by an edge of color $c \in B$ if and only if the corresponding vertices of $\Gamma$ are so (cf. [7]). For standard terminology on graphs see [2].

\subsection{Presentation of Groups}

Given a set $S$, let $F(S)$ denote the free group generated by $S$. So, any element $w$ of $F(S)$ is of the form $w=x_{1}^{\varepsilon_{1}} \cdots x_{m}^{\varepsilon_{m}}$, where $x_{1}, \ldots, x_{m} \in S$ and $\varepsilon_{i}= \pm 1$ for $1 \leqslant i \leqslant m$ and $\left(x_{j+1}, \varepsilon_{j+1}\right) \neq\left(x_{j},-\varepsilon_{j}\right)$ for $1 \leqslant j \leqslant m-1$. For $R \subseteq F(S)$, let $N(R)$ be the smallest normal subgroup of $F(S)$ containing $R$. Then the quotient group $F(S) / N(R)$ is denoted by $\langle S \mid R\rangle$. So, $\langle S \mid T\rangle=\langle S \mid R\rangle$ if $N(T)=N(R)$. We write $\left\langle S_{1} \mid R_{1}\right\rangle=\left\langle S_{2} \mid R_{2}\right\rangle$ only when $F\left(S_{1}\right)=F\left(S_{2}\right)$ and $N\left(R_{1}\right)=N\left(R_{2}\right)$. For $w_{1}, w_{2} \in F(S)$, if $w_{1} N(R)=w_{2} N(R) \in\langle S \mid R\rangle$ then we write $w_{1} \equiv w_{2}(\bmod R)$. Two elements $w_{1}, w_{2} \in F(S)$ are said to be independent (resp., dependent) if $N\left(\left\{w_{1}\right\}\right) \neq N\left(\left\{w_{2}\right\}\right)$ (resp., $N\left(\left\{w_{1}\right\}\right)=N\left(\left\{w_{2}\right\}\right)$ ).

For a finite subset $R$ of $F(S)$, let

$$
\bar{R}:=\{w \in N(R): N((R \backslash\{r\}) \cup\{w\})=N(R) \text { for each } r \in R\} .
$$


Observe that $\bar{\emptyset}=\emptyset$ and if $R \neq \emptyset$ is a finite set then $w:=\prod_{r \in R} r \in \bar{R}$ and hence $\bar{R} \neq \emptyset$. Also, $\left\{w r w^{-1}, w r^{-1} w^{-1}: w \in F(S)\right\} \subseteq \overline{\{r\}}$ for $r \in F(S)$.

For $w=x_{1}^{\varepsilon_{1}} \cdots x_{m}^{\varepsilon_{m}} \in F(S), m \geqslant 1$, let

$$
\varepsilon(w):= \begin{cases}0 & \text { if } m=1 \\ \left|\varepsilon_{1}-\varepsilon_{2}\right|+\cdots+\left|\varepsilon_{m-1}-\varepsilon_{m}\right|+\left|\varepsilon_{m}-\varepsilon_{1}\right| & \text { if } m \geqslant 2 .\end{cases}
$$

Consider the map $\lambda: F(S) \rightarrow \mathbb{Z}^{+}$define inductively as follows.

$$
\lambda(w):= \begin{cases}2 & \text { if } w=\emptyset \\ 2 m-\varepsilon(w) & \text { if } \quad w=x_{1}^{\varepsilon_{1}} \cdots x_{m}^{\varepsilon_{m}},\left(x_{m}, \varepsilon_{m}\right) \neq\left(x_{1},-\varepsilon_{1}\right), \\ \lambda\left(w^{\prime}\right) & \text { if } w=x_{1}^{\varepsilon_{1}} w^{\prime} x_{1}^{-\varepsilon_{1}} .\end{cases}
$$

Since $\left|\varepsilon_{i}-\varepsilon_{j}\right|=0$ or $2, \varepsilon(w)$ is an even integer and hence $\lambda(w)$ is also even. For $w \in F(S)$, $\lambda(w)$ is said to be the weight of $w$. Observe that $\lambda\left(w_{1} w_{2}\right)=\lambda\left(w_{2} w_{1}\right)$ for $w_{1}, w_{2} \in F(S)$.

Let $S=\left\{x_{1}, \ldots, x_{s}\right\}$ and $R=\left\{r_{1}, \ldots, r_{t}\right\} \subseteq F(S)$, where $t \leqslant s$. Let $r_{t+1}$ be an element in $\bar{R}$ of minimum weight. Let

$$
\varphi(S, R):=\lambda\left(r_{1}\right)+\cdots+\lambda\left(r_{t}\right)+\lambda\left(r_{t+1}\right)+2(s-t) .
$$

For a finitely presented group $G$ and a non-negative integer $q$, we define

$$
\mathcal{P}_{q}(G):=\{\langle S \mid R\rangle \cong G: \#(R) \leqslant \#(S) \leqslant q\} .
$$

For a finitely presented group $G$, let $m(G)$ be the minimum number of generators of $G$. Here, we are interested on those groups $G$ for which $\mathcal{P}_{q}(G) \neq \emptyset$ for some $q$. Let

$$
\begin{aligned}
\mu(G) & :=\min \left\{q: \mathcal{P}_{q}(G) \neq \emptyset\right\} \\
\psi(G ; q) & :=\min \left\{\varphi(S, R):\langle S \mid R\rangle \in \mathcal{P}_{q}(G)\right\} \text { for } q \geqslant \mu(G) .
\end{aligned}
$$

Clearly, $\mu(G) \geqslant m(G)$ and $\psi(G, q) \leqslant \psi(G, \mu(G))$ for all $q \geqslant \mu(G)$. Let

$$
\rho(G):=\min \{q \geqslant \mu(G): \psi(G ; q) \leqslant 6(q+1)\} .
$$

So, $\rho(G)$ is the smallest integer $q$ such that $\psi(G ; q) \leqslant 6(q+1)$.

Definition 10. Let $G$ be a group which has a presentation with the number of relations less than or equal to the number of generators. Let $\mu(G), \psi(G ; q)$ and $\rho(G)$ be as above. Then $\psi(G)=\max \{\psi(G ; \rho(G)), 6 \mu(G)+2\}$ is a positive even integer. The integer $\psi(G)$ is said to be the weight of the group $G$.

Remark 11. Observe that $\min \{\varphi(S, R):\langle S \mid R\rangle \cong \mathbb{Z}, \#(R) \leqslant \#(S)<\infty\}=4=$ $\psi(\mathbb{Z}, \rho(\mathbb{Z}))<8=\psi(\mathbb{Z})$ (see the proof of Lemma 3 ). In general, we have $\min \{\varphi(S, R)$ : $\langle S \mid R\rangle \cong G, \#(R) \leqslant \#(S)<\infty\}=\min \left\{\min \left\{\varphi(S, R):\langle S \mid R\rangle \in \mathcal{P}_{q}(G)\right\}: \mu(G) \leqslant q<\right.$ $\infty\}=\min \{\psi(G ; q): \mu(G) \leqslant q<\infty\} \leqslant \psi(G ; \rho(G)) \leqslant \psi(G)$. 


\subsection{Lens Spaces}

Consider the 3 -sphere $S^{3}=\left\{\left(z_{1}, z_{2}\right) \in \mathbb{C}^{2}:\left|z_{1}\right|^{2}+\left|z_{2}\right|^{2}=1\right\}$. Let $p$ and $q$ be relatively prime integers. Then the action of $\mathbb{Z}_{p}=\mathbb{Z} / p \mathbb{Z}$ on $S^{3}$ generated by $e^{2 \pi i / p} \cdot\left(z_{1}, z_{2}\right)=$ $\left(e^{2 \pi i / p} z_{1}, e^{2 \pi i q / p} z_{2}\right)$ is free and hence properly discontinuous. Therefore the quotient space $L(p, q):=S^{3} / \mathbb{Z}_{p}$ is a 3 -manifold whose fundamental group is isomorphic to $\mathbb{Z}_{p}$. The 3 -manifolds $L(p, q)$ are called the lens spaces. It is a classical theorem of Reidemeister that $L\left(p, q^{\prime}\right)$ is homeomorphic to $L(p, q)$ if and only if $q^{\prime} \equiv \pm q^{ \pm 1}(\bmod p)$.

If $T_{1}, T_{2}$ are two solid tori (i.e., each $T_{j}$ is homeomorphic to $\left\{(z, w) \in \mathbb{C}^{2}:|z|=\right.$ $1,|w| \leqslant 1\})$ such that (i) $T_{1} \cap T_{2}=\partial\left(T_{1}\right)=\partial\left(T_{2}\right) \cong S^{1} \times S^{1}$, (ii) $\pi_{1}\left(T_{1} \cap T_{2}, x\right)=$ $\left\langle\alpha, \beta \mid \alpha \beta \alpha^{-1} \beta^{-1}\right\rangle$, (iii) $\pi_{1}\left(T_{1}, x\right)=\langle\alpha\rangle$ and (iv) $\pi_{1}\left(T_{2}, x\right)=\left\langle\alpha, \beta \mid \alpha \beta \alpha^{-1} \beta^{-1}, \alpha^{p} \beta^{q}\right\rangle(=$ $\left\langle\alpha^{m} \beta^{n}\right\rangle$, where $m, n \in \mathbb{Z}$ such that $m q-n p=1$ ), for $x \in T_{1} \cap T_{2}$, then $T_{1} \cup T_{2}$ is homeomorphic to $L(p, q)$.

\subsection{Crystallizations}

A CW-complex $X$ is said to be regular if the attaching maps which define the incidence structure of $X$ are homeomorphisms. Given a regular CW-complex $X$, let $\mathcal{X}$ be the set of all closed cells of $X$ together with the empty set. Then $\mathcal{X}$ is a poset, where the partial ordering is the set inclusion. This poset $\mathcal{X}$ is said to be the face poset of $X$. Clearly, if $X$ and $Y$ are two finite regular $\mathrm{CW}$-complexes with isomorphic face posets then $X$ and $Y$ are homeomorphic. A regular CW-complex $X$ is said to be simplicial if the boundary of each cell in $X$ is isomorphic (as a poset) to the boundary of a simplex of same dimension. A simplicial cell complex $K$ of dimension $d$ is a poset isomorphic to the face poset $\mathcal{X}$ of a $d$-dimensional simplicial CW-complex $X$. The topological space $X$ is called the geometric carrier of $K$ and is also denoted by $|K|$. If a topological space $M$ is homeomorphic to $|K|$, then $K$ is said to be a pseudotriangulation of $M$. A simplicial cell complex $K$ is said to be connected if the topological space $|K|$ is path connected (see $[1,13]$ for more).

Let $K$ be a simplicial cell complex with partial ordering $\leqslant$. If $\beta \leqslant \alpha \in K$ then we say $\beta$ is a face of $\alpha$. For $\alpha \in K$, the set $\partial \alpha:=\{\gamma \in K: \alpha \neq \gamma \leqslant \alpha\}$ is a subcomplex of $K$ with induced partial order and is said to be the boundary of $\alpha$. If $\partial \alpha$ is isomorphic to the boundary complex of an $i$-simplex then we say that $\alpha$ is an $i$-cell or a cell of dimension $i$. For $\beta \in K$, the set $\{\sigma \in K: \beta \leqslant \sigma\}$ is also simplicial cell complex and is said to be the link of $\alpha$ in $K$ and is denoted by $\operatorname{lk}_{K}(\alpha)$.

If all the maximal cells of a $d$-dimensional simplicial cell complex $K$ are $d$-cells then it is called pure. Maximal cells in a pure simplicial cell complex $K$ are called the facets of $K$. Clearly, if $K$ is pure of dimension $d$ and $\alpha$ is an $i$-cell then $\operatorname{lk}_{K}(\alpha)$ is $(d-i-1)$ dimensional and pure. A pure $d$-dimensional simplicial cell complex $K$ is said to be a normal pseudomanifold if each $(d-1)$-cell is a face of exactly two facets and the link of each cell of dimension $\leqslant d-2$ is connected. Clearly, a pseudotriangulation of a connected manifold is a normal pseudomanifold.

The 0 -cells in a simplicial cell complex $K$ are said to be the vertices of $K$. If $u$ is a face of $\alpha$ and $u$ is a vertex then we say $u$ is a vertex of $\alpha$. Clearly, a $d$-dimensional simplicial 
cell complex $\mathcal{X}$ has at least $d+1$ vertices. If a $d$-dimensional simplicial cell complex $\mathcal{X}$ has exactly $d+1$ vertices then $\mathcal{X}$ is called contracted.

Let $\mathcal{X}$ be a pure $d$-dimensional simplicial cell complex. Consider the graph $\Lambda(\mathcal{X})$ whose vertices are the facets of $\mathcal{X}$ and edges are the ordered pairs $\left(\left\{\sigma_{1}, \sigma_{2}\right\}, \gamma\right)$, where $\sigma_{1}, \sigma_{2}$ are facets, $\gamma$ is a $(d-1)$-cell and is a common face of $\sigma_{1}, \sigma_{2}$. The graph $\Lambda(\mathcal{X})$ is said to be the dual graph of $\mathcal{X}$. Observe that $\Lambda(\mathcal{X})$ is in general a multigraph without loops. On the other hand, for $d \geqslant 1$, if $(\Gamma, \gamma)$ is a $(d+1)$-colored graph with color set $C=\{1, \ldots, d+1\}$ then we define a $d$-dimensional simplicial cell complex $\mathcal{K}(\Gamma)$ as follows. For each $v \in V(\Gamma)$ we take a $d$-simplex $\sigma_{v}$ and label its vertices by $1, \ldots, d+1$. If $u, v \in V(\Gamma)$ are joined by an edge $e$ and $\gamma(e)=i$, then we identify the $(d-1)$-faces of $\sigma_{u}$ and $\sigma_{v}$ opposite to the vertices labelled by $i$, so that equally labelled vertices are identified together. Since there is no identification within a $d$-simplex, this gives a simplicial CW-complex $W$ of dimension $d$. So, the face poset (denoted by $\mathcal{K}(\Gamma)$ ) of $W$ is a pure $d$-dimensional simplicial cell complex. We say that $(\Gamma, \gamma)$ represents the simplicial cell complex $\mathcal{K}(\Gamma)$. Clearly, the number of $i$-labelled vertices of $\mathcal{K}(\Gamma)$ is equal to the number of components of $\Gamma_{C \backslash\{i\}}$ for each $i \in C$. Thus, the simplicial cell complex $\mathcal{K}(\Gamma)$ is contracted if and only if $\Gamma$ is contracted (cf. [8]).

A crystallization of a connected closed $d$-manifold $M$ is a $(d+1)$-colored contracted graph $(\Gamma, \gamma)$ such that the simplicial cell complex $\mathcal{K}(\Gamma)$ is a pseudotriangulation of $M$. Thus, if $(\Gamma, \gamma)$ is a crystallization of a $d$-manifold $M$ then the number of vertices in $\mathcal{K}(\Gamma)$ is $d+1$. On the other hand, if $K$ is a contracted pseudotriangulation of $M$ then the dual graph $\Lambda(K)$ gives a crystallization of $M$. Clearly, if $(\Gamma, \gamma)$ is a crystallization of a closed $d$-manifold $M$ then either $\Gamma$ has two vertices (in which case $M$ is $S^{d}$ ) or the number of edges between two vertices is at most $d-1$. From [5], we know the following.

Proposition 12 (Cavicchioli-Grasselli-Pezzana). Let $(\Gamma, \gamma)$ be a crystallization of an $n$ manifold $M$. Then $M$ is orientable if and only if $\Gamma$ is bipartite.

For $k \geqslant 2$, let $1, \ldots, k$ be the colors of a $k$-colored graph $(\Gamma, \gamma)$. For $1 \leqslant i \neq j \leqslant k, \Gamma_{i j}$ denote the graph $\Gamma_{\{i, j\}}$ and $g_{i j}$ denote the number of connected components of the graph $\Gamma_{i j}$. In [9], Gagliardi proved the following.

Proposition 13 (Gagliardi). Let $(\Gamma, \gamma)$ be a contracted 4-colored graph with $m$ vertices. Then $(\Gamma, \gamma)$ is a crystallization of a connected closed 3-manifold if and only if

(i) $g_{i j}=g_{k l}$ for every permutation ijkl of 1234 , and

(ii) $g_{12}+g_{13}+g_{14}=2+m / 2$.

Let $(\Gamma, \gamma)$ be a crystallization (with the color set $C$ ) of a connected closed $n$-manifold $M$. So, $\Gamma$ is an $(n+1)$-regular graph. Choose two colors, say, $i$ and $j$ from $C$. Let $\left\{G_{1}, \ldots, G_{s+1}\right\}$ be the set of all connected components of $\Gamma_{C \backslash\{i, j\}}$ and $\left\{H_{1}, \ldots, H_{t+1}\right\}$ be the set of all connected components of $\Gamma_{i j}$. Since $\Gamma$ is regular, each $H_{p}$ is an even cycle. Note that, if $n=2$, then $\Gamma_{i j}$ is connected and hence $H_{1}=\Gamma_{i j}$. Take a set $\widetilde{S}=\left\{x_{1}, \ldots, x_{s}, x_{s+1}\right\}$ of $s+1$ elements. For $1 \leqslant k \leqslant t+1$, consider the word $\tilde{r}_{k}$ in $F(\widetilde{S})$ 
as follows. Choose a vertex $v_{1}$ in $H_{k}$. Let $H_{k}=v_{1} e_{1}^{i} v_{2} e_{2}^{j} v_{3} e_{3}^{i} v_{4} \cdots e_{2 l-1}^{i} v_{2 l} e_{2 l}^{j} v_{1}$, where $e_{p}^{i}$ and $e_{q}^{j}$ are edges with colors $i$ and $j$ respectively. Define

$$
\tilde{r}_{k}:=x_{k_{2}}^{+1} x_{k_{3}}^{-1} x_{k_{4}}^{+1} \cdots x_{k_{2 l}}^{+1} x_{k_{1}}^{-1},
$$

where $G_{k_{h}}$ is the component of $\Gamma_{C \backslash\{i, j\}}$ containing $v_{h}$. For $1 \leqslant k \leqslant t+1$, let $r_{k}$ be the word obtained from $\tilde{r}_{k}$ by deleting $x_{s+1}^{ \pm 1}$ 's in $\tilde{r}_{k}$. So, $r_{k}$ is a word in $F(S)$, where $S=\widetilde{S} \backslash\left\{x_{s+1}\right\}$. In [10], Gagliardi proved the following.

Proposition 14 (Gagliardi). For $n \geqslant 2$, let $(\Gamma, \gamma)$ be a crystallization of a connected closed n-manifold $M$. For two colors $i, j$, let $s, t, x_{p}, r_{q}$ be as above. If $\pi_{1}(M, x)$ is the fundamental group of $M$ at a point $x$, then

$$
\pi_{1}(M, x) \cong \begin{cases}\left\langle x_{1}, x_{2}, \ldots, x_{s} \mid r_{1}\right\rangle & \text { if } n=2 \\ \left\langle x_{1}, x_{2}, \ldots, x_{s} \mid r_{1}, \ldots, r_{t}\right\rangle & \text { if } n \geqslant 3\end{cases}
$$

\section{Proofs of Lemma 3, Theorem 4 and Corollary 5}

Lemma 3 follows from the next lemma.

Lemma 15. (i) $\psi(\mathbb{Z})=\psi\left(\mathbb{Z}_{2}\right)=8$, (ii) $\psi\left(\mathbb{Z}_{3}\right)=12$, (iii) $\psi\left(\mathbb{Z}_{5}\right)=16$, (iv) $\psi\left(Q_{8}\right)=18$ and $(\mathrm{v}) \psi\left(\mathbb{Z}^{3}\right)=24$.

Proof. Any presentations of $\mathbb{Z}$ must have at least one generator and $\langle x\rangle$ is a presentation of $\mathbb{Z}$. So, $\mu(\mathbb{Z})=1$. If $\langle S \mid R\rangle \cong \mathbb{Z}$ with $\#(S)=1$, then $R=\emptyset$ and hence, by the definition (see $(2.3)), \varphi(S, R)=\lambda(\emptyset)+2(1-0)=2+2=4<12=6(\mu(\mathbb{Z})+1)$. Therefore, $\psi(\mathbb{Z} ; q) \leqslant 4$ for all $q \geqslant 1$. Thus, $\psi(\mathbb{Z})=\max \{\psi(\mathbb{Z}, \rho(\mathbb{Z})), 6 \mu(\mathbb{Z})+2\}=\max \{\psi(\mathbb{Z}, \rho(\mathbb{Z})), 8\}=8$.

Let $p \geqslant 2$ be an integer. Since any presentations of $\mathbb{Z}_{p}$ must have at least one generator and $\left\langle x \mid x^{p}\right\rangle$ is a presentation of $\mathbb{Z}_{p}$, it follows that $\mu\left(\mathbb{Z}_{p}\right)=1$. Clearly, if $\langle S=\{x\}| R=$ $\left.\left\{r_{1}\right\}\right\rangle$ is a presentation of $\mathbb{Z}_{p}$, then $r_{1}=x^{ \pm p}$. Let $r_{2} \in \bar{R}$ be of minimum weight. Since $\left\langle x \mid r_{2}\right\rangle$ is also a presentation of $\mathbb{Z}_{p}, r_{2}=x^{ \pm p}$. Therefore, by (2.3),

$$
\varphi(S, R)=\lambda\left(r_{1}\right)+\lambda\left(r_{2}\right)=\left(2 p-\varepsilon\left(r_{1}\right)\right)+\left(2 p-\varepsilon\left(r_{2}\right)\right)=4 p .
$$

First assume that $p \leqslant 3$. Since, $\langle S \mid R\rangle \in \mathcal{P}_{1}\left(\mathbb{Z}_{p}\right)$ implies (up to renaming) $(S, R)=$ $\left(\{x\},\left\{x^{p}\right\}\right)$ or $\left(\{x\},\left\{x^{-p}\right\}\right)$, it follows that $\psi\left(\mathbb{Z}_{p} ; 1\right)=\varphi\left(\{x\},\left\{x^{ \pm p}\right\}\right)=4 p \leqslant 12=$ $6\left(\mu\left(\mathbb{Z}_{p}\right)+1\right)$. This implies that $\rho\left(\mathbb{Z}_{p}\right)=\mu\left(\mathbb{Z}_{p}\right)=1$. Thus, $\psi\left(\mathbb{Z}_{p} ; \rho\left(\mathbb{Z}_{p}\right)\right)=4 p \geqslant 8=$ $6 \mu\left(\mathbb{Z}_{p}\right)+2$. Therefore, $\psi\left(\mathbb{Z}_{p}\right)=4 p$. This proves parts (i) and (ii).

Now, assume $p=5$. By the similar arguments as for $p \leqslant 3,\langle S \mid R\rangle \in \mathcal{P}_{1}\left(\mathbb{Z}_{5}\right)$ implies $\varphi(S, R)=4 p=20$. Therefore, $\psi\left(\mathbb{Z}_{5} ; 1\right)=20>12=6\left(\mu\left(\mathbb{Z}_{5}\right)+1\right)$ and hence $\rho\left(\mathbb{Z}_{5}\right)>$ $\mu\left(\mathbb{Z}_{5}\right)=1$. If we take $S=\left\{x_{1}, x_{2}\right\}$ and $R=\left\{r_{1}=x_{1}^{2} x_{2}^{-1}, r_{2}=x_{2}^{3} x_{1}^{-1}\right\}$ then $\varphi(S, R) \leqslant 16$ (since $r_{3}=x_{1} x_{2}^{2} \in \bar{R}$ is of weight 6 ) and $\langle S \mid R\rangle \in \mathcal{P}_{2}\left(\mathbb{Z}_{5}\right) \backslash \mathcal{P}_{1}\left(\mathbb{Z}_{5}\right)$. Thus, $\psi\left(\mathbb{Z}_{5} ; 2\right) \leqslant 16<$ $18=6(2+1)$. Therefore, $\rho\left(\mathbb{Z}_{5}\right)=2$ and hence $\psi\left(\mathbb{Z}_{5}\right) \leqslant 16$.

Now, let $\langle S \mid R\rangle \in \mathcal{P}_{2}\left(\mathbb{Z}_{5}\right) \backslash \mathcal{P}_{1}\left(\mathbb{Z}_{5}\right)$ with $\varphi(S, R) \leqslant 16$. Since there is no presentation $\langle S \mid R\rangle$ of $\mathbb{Z}_{5}$ with $(\#(S), \#(R))=(2,1)$, it follows that $\#(R)=\#(S)=2$. Let $S=$ 
$\left\{x_{1}, x_{2}\right\}$ and $R=\left\{r_{1}, r_{2}\right\}$. If $\lambda\left(r_{1}\right)=2$, then $r_{1}$ must be of the form $x_{i}^{ \pm 1}$ or $x_{i}^{\varepsilon} x_{j}^{-\varepsilon}$ for some $j \neq i \in\{1,2\}$ and $\varepsilon= \pm 1$. Since $\langle S \mid R\rangle \cong \mathbb{Z}_{5}$, it follows that $r_{2} \equiv x_{j}^{ \pm 5}(\bmod$ $\left.\left\{r_{1}\right\}\right)$. This implies that $\lambda\left(r_{2}\right) \geqslant \lambda\left(x_{j}^{ \pm 5}\right)=10$. Let $r_{3} \in \bar{R}$ be of minimum weight. Then $\left\langle x_{1}, x_{2} \mid r_{1}, r_{3}\right\rangle$ is also a presentation of $\mathbb{Z}_{5}$ and hence (by the same arguments) $\lambda\left(r_{3}\right) \geqslant 10$. Thus, $\varphi(S, R)=\lambda\left(r_{1}\right)+\lambda\left(r_{2}\right)+\lambda\left(r_{3}\right) \geqslant 2+10+10=22$, a contradiction. So, $\lambda\left(r_{i}\right) \geqslant 4$ for $1 \leqslant i \leqslant 2$. Let $A=\left\{x_{1} x_{2}, x_{1}^{2}, x_{2}^{2}, x_{1}^{2} x_{2}^{-1}, x_{2}^{2} x_{1}^{-1}, x_{1} x_{2}^{-1} x_{1} x_{2}^{-1}\right\}$ and let $A^{-1}=\left\{w^{-1}: w \in A\right\}$. Then $A$ is a set of pairwise independent elements of weight 4 in $F(S)$ and $w \in F(S)$ is an element of weight 4 imply that $w$ is dependent with an element of $A$. Note that $\mathbb{Z}_{5}$ has no presentation $\langle S \mid R\rangle \in \mathcal{P}_{2}\left(\mathbb{Z}_{5}\right) \backslash \mathcal{P}_{1}\left(\mathbb{Z}_{5}\right)$ with $R \subseteq A \cup A^{-1}$. So, at most one of $r_{1}, r_{2}, r_{3}$ has weight 4 and the weights of other two are at least 6 . Therefore, $\varphi(S, R) \geqslant 16$. This implies that $\psi\left(\mathbb{Z}_{5}\right)=16$. This proves part (iii).

Clearly, $\mu\left(Q_{8}\right)=2$. If we take $S=\left\{x_{1}, x_{2}\right\}$ and $R=\left\{x_{2} x_{1} x_{2} x_{1}^{-1}, x_{1} x_{2} x_{1} x_{2}^{-1}\right\}$ then $\langle S \mid R\rangle \in \mathcal{P}_{2}\left(Q_{8}\right)$ and $\varphi(S, R) \leqslant 18$ (since $x_{2}^{2} x_{1}^{-2} \in \bar{R}$ is of weight 6 ). Thus $\psi\left(Q_{8} ; 2\right) \leqslant$ $18=6(2+1)$. Therefore, $\rho\left(Q_{8}\right)=2$ and hence $\psi\left(Q_{8}\right) \leqslant 18$.

Now, let $\varphi(S, R) \leqslant 18$, where $S=\left\{x_{1}, x_{2}\right\}$ and $\langle S \mid R\rangle \in \mathcal{P}_{2}\left(Q_{8}\right)$. Note that $B=\left\{x_{1} x_{2}, x_{1}^{2}, x_{2}^{2}, x_{1}^{2} x_{2}^{-1}, x_{2}^{2} x_{1}^{-1}, x_{1} x_{2}^{-1} x_{1} x_{2}^{-1}, x_{2}^{2} x_{1}, x_{1}^{3} x_{2}^{-1}, x_{2}^{2} x_{1}^{-1} x_{2} x_{1}^{-1}, x_{1}^{2} x_{2}, x_{2}^{3} x_{1}^{-1}, x_{1}^{3}, x_{2}^{3}\right.$, $\left.x_{1}^{2} x_{2}^{-1} x_{1} x_{2}^{-1}, x_{1} x_{2} x_{1} x_{2}^{-1}, x_{2} x_{1} x_{2} x_{1}^{-1}, x_{1} x_{2}^{-1} x_{1} x_{2}^{-1} x_{1} x_{2}^{-1}, x_{1} x_{2} x_{1}^{-1} x_{2}^{-1}, x_{2}^{2} x_{1}^{-2}\right\}$ is a set of pairwise independent elements of weight 4 or 6 in $F(S)$. It is not difficult to see that $w \in F(S)$ and $4 \leqslant \lambda(w) \leqslant 6$ imply $w$ is dependent with an element of $B$. Let $B^{-1}=\left\{w^{-1}: w \in B\right\}$. Then $R \subseteq B \cup B^{-1}$. Clearly, the only possible choices of $\left\{r_{1}^{ \pm 1}, r_{2}^{ \pm 1}\right\}$ are $\left\{x_{2}^{2} x_{1}^{-2}, x_{1} x_{2} x_{1} x_{2}^{-1}\right\}$, $\left\{x_{2}^{2} x_{1}^{-2}, x_{2} x_{1} x_{2} x_{1}^{-1}\right\}$ and $\left\{x_{2} x_{1} x_{2} x_{1}^{-1}, x_{1} x_{2} x_{1} x_{2}^{-1}\right\}$. Then $\lambda(r) \geqslant 6$ for $r \in R \cup \bar{R}$. Thus, $\varphi(S, R) \geqslant 18$. Therefore, $\psi\left(Q_{8}\right)=18$. This proves parts (iv).

Clearly, $\mu\left(\mathbb{Z}^{3}\right)=3$. If $S_{0}=\left\{x_{1}, x_{2}, x_{3}\right\}$ and $R_{0}=\left\{x_{i} x_{j} x_{i}^{-1} x_{j}^{-1}: 1 \leqslant i<j \leqslant 3\right\}$ then $\left\langle S_{0} \mid R_{0}\right\rangle \in \mathcal{P}_{3}\left(\mathbb{Z}^{3}\right)$ and $\varphi\left(S_{0}, R_{0}\right) \leqslant 24$ (since $x_{1} x_{2}^{-1} x_{3} x_{1}^{-1} x_{2} x_{3}^{-1} \in \bar{R}_{0}$ is of weight 6 ). Thus $\psi\left(\mathbb{Z}^{3} ; 3\right) \leqslant 24=6(3+1)$. Therefore, $\rho\left(\mathbb{Z}^{3}\right)=3$ and hence $\psi\left(\mathbb{Z}^{3}\right) \leqslant 24$.

Claim. If $w \in N\left(R_{0}\right)$ is not the identity then $\lambda(w) \geqslant 6$.

If $w \in N\left(R_{0}\right)$ is not the identity then clearly $\lambda(w) \neq 2$. Observe that, if $w \in F\left(S_{0}\right)$ with $\lambda(w)=4$, then $w$ is dependent with an element of the set $C=\left\{x_{i}^{2} x_{j}^{-1}, x_{i} x_{j}^{-1} x_{i} x_{j}^{-1}, x_{i}^{2}, x_{i} x_{j}\right.$, $x_{i} x_{j}^{-1} x_{i} x_{k}^{-1}: i j k$ is a permutation of 123$\}$. Since none of the element in $C$ is in $N\left(R_{0}\right)$, it follows that $N\left(R_{0}\right)$ has no element of weight 4 . This proves the claim.

Now, let $\varphi(S, R) \leqslant 24$, where $S=\left\{x_{1}, x_{2}, x_{3}\right\}$ and $\langle S \mid R\rangle \in \mathcal{P}_{3}\left(\mathbb{Z}^{3}\right)$. Then $N(R)=$ $N\left(R_{0}\right)$ and hence, by the claim, weight of each element of $R$ is at least 6 . This implies $\varphi(S, R) \geqslant 24$ and hence $\varphi(S, R)=24$. Therefore, $\psi\left(\mathbb{Z}^{3}\right)=24$. This completes the proof.

Proof of Theorem 4. Let $G=\pi(M, x)$ for some $x \in M$. To prove the theorem, it is sufficient to show that any crystallization of $M$ needs at least $\psi(M)=\psi(G)$ vertices.

Let $(\Gamma, \gamma)$ be a crystallization of $M$ with $m$ vertices and let $\{1,2,3,4\}$ be the color set. Then, by Proposition 14, we know that $G$ has a presentation with $g_{i j}-1$ generators and $\leqslant g_{i j}-1$ relations. Therefore, by the definition of $\mu(G)$ (in $\left.(2.4 \mathrm{a})\right), \mu(G) \leqslant g_{i j}-1$. Then, by part (ii) of Proposition 13,

$$
m=2\left(g_{12}+g_{13}+g_{14}\right)-4 \geqslant 6(\mu(G)+1)-4=6 \mu(G)+2 .
$$


From the definition of $\rho(G)$ (in (2.5)), $6(\rho(G)+1) \geqslant \psi(G ; \rho(G)$ ). Therefore, $m>$ $6(\rho(G)+1)$ implies $m>\psi(G ; \rho(G))$. Thus, if $m>6(\rho(G)+1)$ then the result follows from this and Eq. (3.2).

Now, assume that $m \leqslant 6(\rho(G)+1)$. Then, by part (ii) of Proposition $13, g_{12}+g_{13}+$ $g_{14} \leqslant 2+3(\rho(G)+1)$. This implies, $g_{1 j} \leqslant \rho(G)+1$ for some $j \in\{2,3,4\}$. Assume, without loss, that $g_{12} \leqslant \rho(G)+1$.

As in Subsection 2.4, let $G_{1}, \ldots, G_{q+1}$ be the components of $\Gamma_{12}$ and $H_{1}, \ldots, H_{q+1}$ be the components of $\Gamma_{34}$, where $q+1=g_{34}=g_{12} \leqslant \rho(G)+1$. By Proposition $14, G$ has a presentation of the form $\left\langle x_{1}, x_{2}, \ldots, x_{q} \mid r_{1}, r_{2}, \ldots, r_{q}\right\rangle$, where $x_{k}$ corresponds to $G_{k}$ and $r_{k}$ corresponds to $H_{k}$ as in Subsection 2.4. Let $S=\left\{x_{1}, x_{2}, \ldots, x_{q}\right\}$ and $R=\left\{r_{1}, \ldots, r_{q}\right\}$.

For $1 \leqslant i \leqslant q$, let $r_{i}=x_{i_{1}}^{\varepsilon_{1}} \cdots x_{i_{n}}^{\varepsilon_{n}}$, where $x_{i_{1}}, \ldots, x_{i_{n}} \in\left\{x_{1}, \ldots, x_{q}\right\}$ and $\varepsilon_{j}= \pm 1$ for $1 \leqslant j \leqslant n,\left(x_{i_{j+1}}, \varepsilon_{j+1}\right) \neq\left(x_{i_{j}},-\varepsilon_{j}\right)$ for $1 \leqslant j \leqslant n-1$ and $\left(x_{i_{n}}, \varepsilon_{n}\right) \neq\left(x_{i_{1}},-\varepsilon_{1}\right)$.

Claim. For $1 \leqslant i \leqslant q$, the length of the cycle $H_{i}$ is at least $\lambda\left(r_{i}\right)$.

Consider the word $\tilde{r}_{i}$ (in $F\left(\left\{x_{1}, \ldots, x_{q}, x_{q+1}\right\}\right)$ which is obtained from $r_{i}$ by the following rules: if $\varepsilon_{j}=\varepsilon_{j+1}$ for $1 \leqslant j \leqslant n-1$, then replace $x_{i_{j}}^{\varepsilon_{j}}$ by $x_{i_{j}}^{\varepsilon_{j}} x_{q+1}^{-\varepsilon_{j}}$ in $r_{i}$ and if $\varepsilon_{n}=\varepsilon_{1}$, then replace $x_{i_{n}}^{\varepsilon_{n}}$ by $x_{i_{n}}^{\varepsilon_{n}} x_{q+1}^{-\varepsilon_{n}}$ in $r_{i}$. Observe that $\tilde{r}_{i}$ is non empty (since $r_{i}$ is non empty) and the number of letters in $\tilde{r}_{i}$ is same as $\lambda\left(r_{i}\right)$ (see (2.6) and (2.2)). The claim follows from this.

Let $r_{q+1}$ be a word corresponding to $H_{q+1}$ in $\Gamma_{34}$. Then, any $q$ of the relations from the set $\left\{r_{1}, r_{2}, \ldots, r_{q}, r_{q+1}\right\}$ together with the generators $x_{1}, x_{2}, \ldots, x_{q}$ give a presentation of $G$. This implies, $r_{q+1} \in \bar{R}$. Thus, $m \geqslant \lambda\left(r_{1}\right)+\lambda\left(r_{2}\right)+\cdots+\lambda\left(r_{q+1}\right) \geqslant \varphi(S, R) \geqslant \psi(G ; \rho(G))$. Therefore, $m \geqslant \max \{\psi(G ; \rho(G)), 6 a(G)+2\}=\psi(G)$. This proves the theorem.

Proof of Corollary 5. Let $f_{i}$ be the number of $i$-cells in $X$. So, $f_{0}=4$. Therefore, $g_{2}(X)=f_{1}-16+10=f_{1}-6=f_{1}-12+6=h_{2}(X)$. Since $|X|$ is a closed 3-manifold, each 2-cell is a face of two 3 -cells and each 3 -cell has four 2-dimensional faces. This implies that $2 f_{2}=4 f_{3}$. Then, $0=f_{0}-f_{1}+f_{2}-f_{3}=4-f_{1}+2 f_{3}-f_{3}$. Thus, $f_{1}=f_{3}+4$ and hence $g_{2}(X)=h_{2}(X)=f_{3}-2$. Therefore, by Theorem $4, g_{2}(X)=h_{2}(X)=f_{3}-2 \geqslant$ $\Psi(M)-2 \geqslant \psi(M)-2$.

From the definition of $\psi(G), \psi(G) \geqslant 6 \mu(G)+2 \geqslant 6 m(G)+2$. So, $\psi(M)=\psi(\pi(M, *))$ $\geqslant 6 m(\pi(M, *))+2$. Since any presentation of $\pi(M, *)$ has at least $\beta_{1}(M ; \mathbb{F})$ generators, it follows that $m(M)=m(\pi(M, *)) \geqslant \beta_{1}(M ; \mathbb{F})$. The corollary now follows.

Remark 16. If a crystallization $(\Gamma, \gamma)$ yields a presentation $\langle S \mid R\rangle$ then, from the proof of Theorem 4 , we get $\varphi(S, R) \leqslant$ the number of vertices of $\Gamma$.

Remark 17. We found that $\rho\left(\mathbb{Z}^{3}\right)=3$ and $\varphi(S, R)=24$, where $\langle S \mid R\rangle \in \mathcal{P}_{3}\left(\mathbb{Z}^{3}\right)$. On the other hand, if $S^{\prime}=\left\{x_{1}, \ldots, x_{5}\right\}$ and $R^{\prime}=\left\{x_{1} x_{4}^{-1} x_{5} x_{3}^{-1}, x_{1} x_{5} x_{2}^{-1}, x_{3} x_{4} x_{2}^{-1}, x_{1} x_{3}^{-1} x_{5} x_{4}^{-1}\right.$, $\left.x_{5} x_{1} x_{2}^{-1}\right\}$ then $\left\langle S^{\prime} \mid R^{\prime}\right\rangle \in \mathcal{P}_{5}\left(\mathbb{Z}^{3}\right) \backslash \mathcal{P}_{4}\left(\mathbb{Z}^{3}\right)$ with $\varphi\left(S^{\prime}, R^{\prime}\right)=24$. So, the minimum weight presentation of $\mathbb{Z}^{3}$ is not unique. This is true for most of the groups. 


\section{Uniqueness of some crystallizations}

Here, we are interested on crystallizations of 3-manifolds $M$ with $\psi(M)$ vertices. For seven 3-manifolds, we show that there exists a unique such crystallization for each of them.

Throughout this section and behind, 1,2,3,4 are the colors of a 4-colored graph $(\Gamma, \gamma)$ and $g_{i j}$ is the number of components of $\Gamma_{i j}=\Gamma_{\{i, j\}}$ for $i \neq j$.

Let $\mathcal{X}$ be the pseudotriangulation of a connected closed 3-manifold $M$ determined by a crystallization $(\Gamma, \gamma)$. So, $(\Gamma, \gamma)$ is contracted, i.e., $\Gamma_{\{i, j, k\}}$ is connected for $i, j, k$ distinct. For $1 \leqslant i \leqslant 4$, we denote the vertex of $\mathcal{X}$ corresponding to the color $i$ by $v_{i}$. We identify a vertex $u$ of $\Gamma$ with the corresponding facet $\sigma_{u}$ of $\mathcal{X}$. For a facet $u\left(\equiv \sigma_{u}\right)$ of $\mathcal{X}$, the 2 -face of $u$ not containing the vertex $v_{i}$ will be denoted by $u_{i}$. Similarly, the edge of $u$ not containing the vertices $v_{i}, v_{j}$ will be denoted by $u_{i j}$. Clearly, if $C_{2 k}\left(u^{1}, u^{2}, \ldots, u^{2 k}\right)$ is a $2 k$-cycle in $\Gamma$ with colors $i$ and $j$ alternately, then $u_{i j}^{1}=u_{i j}^{2}=\cdots=u_{i j}^{2 k}$ in $\mathcal{X}$.

Lemma 18. Let $\Gamma$ be a crystallization of a connected closed 3-manifold $M$ with $m$ vertices. If $\Gamma$ has a 2-cycle, then either $M$ has a crystallization with $m-2$ vertices or $\pi_{1}(M, x)$ $($ for $x \in M)$ is isomorphic to the free product $\mathbb{Z} * H$ for some group $H$.

Proof. Without loss of generality, assume that $\Gamma$ has a 2-cycle with color 1 and 2, i.e., $\Gamma_{12}$ has a component of length 2. If this 2-cycle touches two different components of $\Gamma_{34}$ (say, at vertices $v$ and $w$, respectively), then $\Gamma[\{v, w\}]$ is a 3-dimensional dipole of type 2 . Therefore, the crystallization $\Gamma$ can be reduced to a crystallization $\Gamma^{1}$ of $M$ with vertex set $V(\Gamma) \backslash\{v, w\}$ so that $\Gamma_{12}^{1}$ (resp., $\Gamma_{34}^{1}$ ) has one less components than $\Gamma_{12}$ (resp., $\Gamma_{34}$ ) as in Fig. 1 (see [7]). Thus, $M$ has a crystallization (namely, $\Gamma^{1}$ ) with $m-2$ vertices.

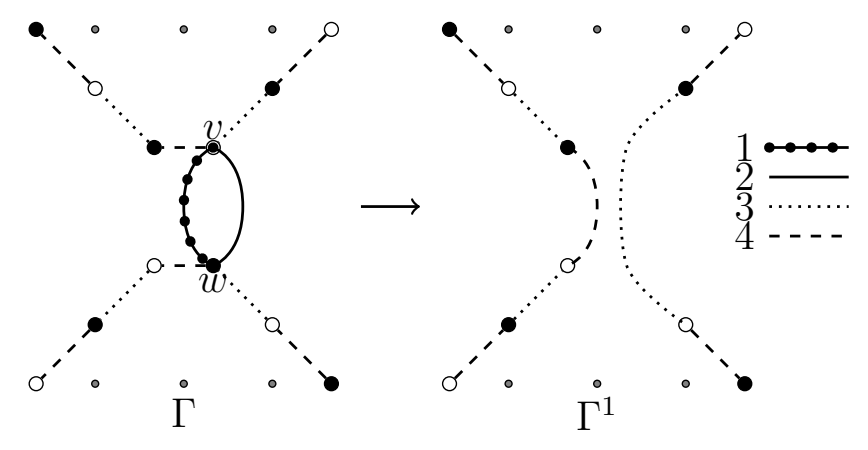

Figure 1: Cancellation of a dipole of type 2

So, assume that the 2-cycle (say $G_{1}$ ) touches only one component (say, $H_{1}$ ) of $\Gamma_{34}$. Let $G_{1}, \ldots, G_{q+1}$ be the components of $\Gamma_{12}$ and $H_{1}, \ldots, H_{q+1}$ be the components of $\Gamma_{34}$, where $q+1=g_{12}=g_{34}$. Let $x_{1}, \ldots, x_{q+1}$ and $r_{1}, \ldots, r_{q+1}$ be as in Proposition 14 . Then, by Proposition 14, $\pi_{1}(M, x)$ has a presentation of the form $\left\langle x_{1}, x_{2}, \ldots, x_{q} \mid r_{2}, r_{3}, \ldots, r_{q+1}\right\rangle$. Since $G_{1}$ touches only $H_{1}$, from the definition of $\tilde{r}_{k}$ in Eq. (2.6), $\tilde{r}_{k}$ does not contain $x_{1}^{ \pm 1}$ for $k \neq 1$. Therefore, $\left\langle x_{1}, x_{2}, \ldots, x_{q} \mid r_{2}, \ldots, r_{q+1}\right\rangle=\left\langle x_{1}\right\rangle *\left\langle x_{2}, \ldots, x_{q} \mid r_{2}, \ldots, r_{q+1}\right\rangle$. This proves the lemma. 
Lemma 19. There exist exactly three 8-vertex crystallizations of non-simply connected, connected, closed 3-manifolds. Moreover, these three are crystallizations of $S^{2} \times S^{1}$, $S^{2} \times S^{1}$ and $\mathbb{R} \mathbb{P}^{3}$ respectively.

Proof. Let $(\Gamma, \gamma)$ be an 8-vertex crystallization of a non simply connected, connected, closed 3-manifold $M$. By Proposition 13, $g_{12}+g_{13}+g_{14}=8 / 2+2=6$ and $g_{i j}=g_{k l}$ for $i, j, k, l$ distinct. Since $\pi_{1}(M, *)$ has at least one generator, $g_{i j} \geqslant 2$ for $1 \leqslant i \neq j \leqslant 4$. This implies that $g_{i j}=2$ and hence $\Gamma_{i j}$ is of the form $C_{2} \sqcup C_{6}$ or $C_{4} \sqcup C_{4}$ for $1 \leqslant i \neq j \leqslant 4$.

Case 1: Suppose $(\Gamma, \gamma)$ has a 2-cycle. Since $M$ is not simply connected, $M$ has no crystallization with less than 8 vertices. Therefore, by Lemma $18, \pi_{1}(M, *)$ must have a torsion free element. Again, $g_{i j}=2$ implies $\pi_{1}(M, *)$ is generated by one element and hence isomorphic to $\mathbb{Z}$. Therefore, $M \cong S^{2} \times S^{1}$ or $S^{2} \times S^{1}$. Assume, without loss, $\Gamma_{12}=G_{1} \sqcup G_{2}$, where $G_{1}=C_{2}\left(v_{3}, v_{4}\right), G_{2}=C_{6}\left(v_{1}, v_{2}, v_{5}, v_{6}, v_{7}, v_{8}\right)$. Then there is no edge between $v_{3}$ and $v_{4}$ of color 3 or 4 and (see the proof of Lemma 18), $G_{1}$ touches only one component of $\Gamma_{34}$. Let $\Gamma_{34}=H_{1} \sqcup H_{2}$, where $G_{1} \cap H_{1}=\emptyset$. Let $x$ and $y$ be the generators corresponding to the components $G_{1}$ and $G_{2}$ respectively. If $H_{2}$ is a 4-cycle then $\mathrm{H}_{2}$ represents $x y^{-1} x y^{-1}$ by choosing some $v_{1}, i, j$ as in Eq. (2.6). But $x y^{-1} x y^{-1}$ does not give identity relation by deleting $x$ or $y$. Therefore, $H_{2}$ is a 6-cycle and hence $H_{1}$ is a 2-cycle. Similarly, $G_{2} \cap H_{2}=\emptyset$. Since the number of edges between any pair of vertices is at most 2 , we can assume that $H_{1}=C_{2}\left(v_{1}, v_{6}\right)$. Assume, without loss, that there is an edge of color 4 between $v_{2}$ and $v_{3}$. Since $\Gamma_{24}$ has two components, this implies $\Gamma_{24}=C_{4}\left(v_{4}, v_{3}, v_{2}, v_{5}\right) \sqcup C_{4}\left(v_{8}, v_{1}, v_{6}, v_{7}\right)$. So, there exists an edge of color 4 between $v_{4}$ and $v_{5}$ (resp. $v_{7}$ and $v_{8}$ ). Since $H_{2}$ is a 6-cycle on the vertex set $\left\{v_{1}, \ldots, v_{8}\right\} \backslash\left\{v_{1}, v_{6}\right\}$, this implies that $H_{2}=C_{6}\left(v_{2}, v_{3}, v_{8}, v_{7}, v_{4}, v_{5}\right)$ or $C_{6}\left(v_{2}, v_{3}, v_{7}, v_{8}, v_{4}, v_{5}\right)$. In the first case, $(\Gamma, \gamma)=\mathcal{J}_{1}$ and in the second case, $(\Gamma, \gamma)=\mathcal{J}_{2}$ given in Fig. 2 (a) and (b) respectively. In the first case, $\Gamma$ is bipartite. Therefore, $M$ is orientable and hence equal to $S^{2} \times S^{1}$. In the second case, $\Gamma$ is not bipartite. Therefore, $M$ is non-orientable and hence equal to $S^{2} \times S^{1}$.

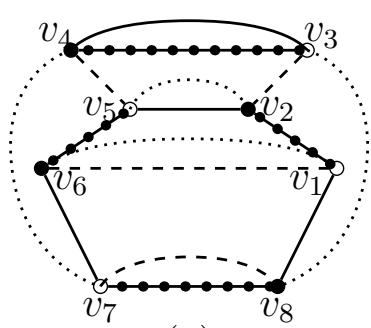

(a)

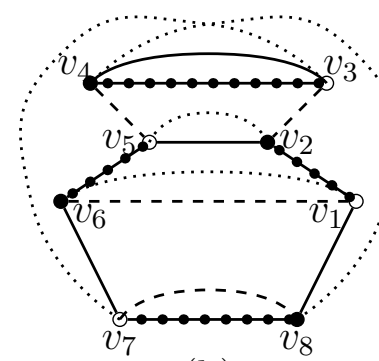

(b)

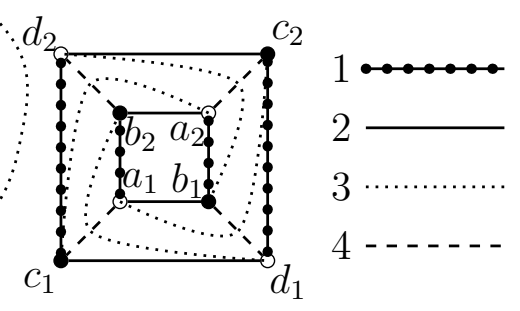

(c)

Figure 2: Crystallizations $\mathcal{J}_{1}, \mathcal{J}_{2}$ and $\mathcal{K}_{2,1}$

Case 2: Suppose $(\Gamma, \gamma)$ has no 2-cycle. So, $\Gamma$ is a simple graph. Then, $\Gamma_{i j}=C_{4} \sqcup C_{4}$ for $1 \leqslant i \neq j \leqslant 4$. Let $G_{1}=C_{4}\left(a_{1}, b_{1}, a_{2}, b_{2}\right)$ and $G_{2}=C_{4}\left(c_{1}, d_{1}, c_{2}, d_{2}\right)$ be the components of $\Gamma_{12}$. If $a_{1} a_{2}$ is an edge of color 3 then (since $\Gamma_{13}=C_{4} \sqcup C_{4}$ ) $b_{1} b_{2}$ must be an edge of color 3. Then $\Gamma_{123}$ is disconnected. This is not possible. So, $a_{1} a_{2}$ cannot be an edge of color 3 . 
Similarly, $a_{1} a_{2}$ cannot be an edge of color 4 . These imply, $a_{1} a_{2}$ can not be an edge of $\Gamma$. Assume, without loss, $a_{1} c_{1}$ is an edge of color 4 . Then $a_{2} c_{2}, b_{1} d_{1}, b_{2} d_{2}$ are edges of color 4 (since $\Gamma_{i 4}=C_{4} \sqcup C_{4}$ for $1 \leqslant i \leqslant 2$ ). If $a_{1} d_{1}$ is an edge of color 3 , then $C_{4}\left(b_{1}, a_{1}, d_{1}, c_{1}\right)$ would be a component of $\Gamma_{23}$. This implies $\Gamma\left[\left\{a_{1}, b_{1}, c_{1}, d_{1}\right\}\right]$ would be proper component of $\Gamma_{\{2,3,4\}}$. This is not possible since $(\Gamma, \gamma)$ is a contracted graph. Thus, $a_{1} d_{1}$ is not an edge of color 3. Similarly, $a_{1} d_{2}$ is not an edge of color 3 . These imply $a_{1} c_{2}$ is an edge of color 3. Similarly, $b_{1} d_{2}, a_{2} c_{1}$ and $b_{2} d_{1}$ are edges of color 3. Then, $(\Gamma, \gamma)=\mathcal{K}_{2,1}$ given in Fig. 2 (c). Since $G_{1}=C_{4}\left(a_{1}, b_{1}, a_{2}, b_{2}\right)$ and $H_{1}=C_{4}\left(d_{1}, b_{2}, d_{2}, b_{1}\right)$ is a component of $\Gamma_{34}$, $\pi(M, *)=\left\langle x \mid x^{2}\right\rangle \cong \mathbb{Z}_{2}$. This implies that $M=\mathbb{R P}^{3}$. This completes the proof.

Lemma 20. There exists a unique 12-vertex crystallization of $L(3,1)$.

Proof. By Lemma 15 and Theorem 4, L(3,1) has no crystallization with less than 12 vertices. Let $(\Gamma, \gamma)$ be a 12 -vertex crystallization of $L(3,1)$. Since $\pi_{1}(L(3,1), *)\left(\cong \mathbb{Z}_{3}\right)$ has no torsion free element, by Lemma 18, $(\Gamma, \gamma)$ has no 2-cycle. So, $\Gamma$ is a simple graph. This implies that $g_{i j} \leqslant 3$ for $i \neq j$. Also (since $\mathbb{Z}_{3}$ has at least one generator) $g_{i j} \geqslant 2$. By Proposition 13, $g_{12}+g_{13}+g_{14}=12 / 2+2=8$ and $g_{i j}=g_{k l}$ for $i, j, k, l$ distinct. So, without loss, we can assume that $g_{12}=g_{34}=2, g_{13}=g_{14}=3$. Then $\Gamma_{i j}=C_{4} \sqcup C_{4} \sqcup C_{4}$ for $1 \leqslant i \leqslant 2,3 \leqslant j \leqslant 4$. Let $G_{1}, G_{2}$ be the components of $\Gamma_{12}$ and $H_{1}, H_{2}$ be the components of $\Gamma_{34}$ such that $x_{1}, x_{2}$ represent the generators corresponding to $G_{1}, G_{2}$ respectively. Since $\left\langle x_{j} \mid x_{j}^{3}\right\rangle$ is the only presentation in $\mathcal{P}_{1}\left(\mathbb{Z}_{3}\right), H_{i}$ must yield the relations $x_{j}^{ \pm 3}$, for $1 \leqslant i, j \leqslant 2$. Therefore, $G_{i}$ and $H_{i}$ are 6 -cycles. Let $G_{1}=C_{6}\left(a_{1}, b_{1}, \ldots, a_{3}, b_{3}\right)$ and $G_{2}=C_{6}\left(c_{1}, d_{1}, \ldots, c_{3}, d_{3}\right)$. Assume, without loss, $a_{1} c_{1} \in \gamma^{-1}(4)$. Then $C_{4}\left(b_{3}, a_{1}, c_{1}, d_{3}\right) \subseteq$ $\Gamma_{14}$ and hence $b_{3} d_{3} \in \gamma^{-1}(4)$. Similarly, $a_{3} c_{3}, b_{2} d_{2}, a_{2} c_{2}, b_{1} d_{1} \in \gamma^{-1}(4)$. Now, $a_{1} d_{1} \in \gamma^{-1}(3)$ $\Longrightarrow C_{4}\left(a_{1}, d_{1}, c_{1}, b_{1}\right) \subseteq \Gamma_{23} \Longrightarrow \Gamma\left[\left\{a_{1}, b_{1}, c_{1}, d_{1}\right\}\right]$ is a component of $\Gamma_{\{2,3,4\}}$. This is not possible since $\Gamma$ is a contracted graph. So, $a_{1} d_{1} \notin \gamma^{-1}(3)$. Similarly, $a_{1} d_{3} \notin \gamma^{-1}(3)$. Again, $a_{1} d_{2} \in \gamma^{-1}(3) \Longrightarrow C_{4}\left(a_{1}, d_{2}, c_{2}, b_{1}\right) \subseteq \Gamma_{23} \Longrightarrow c_{2} b_{1} \in \gamma^{-1}(3) \Longrightarrow C_{4}\left(a_{2}, b_{1}, c_{2}, d_{1}\right) \subseteq \Gamma_{13} \Longrightarrow$ $\Gamma\left[\left\{a_{2}, b_{1}, c_{2}, d_{1}\right\}\right]$ is a component of $\Gamma_{\{1,3,4\}}$, a contradiction. So, $a_{1} d_{2} \notin \gamma^{-1}(3)$. Therefore, up to an isomorphism, $a_{1} c_{2} \in \gamma^{-1}(3)$. Then $b_{1} d_{2}, a_{2} c_{3}, b_{2} d_{3}, a_{3} c_{1}, b_{3} d_{1} \in \gamma^{-1}(3)$ and hence $(\Gamma, \gamma)=\mathcal{K}_{3,1}$ given in Fig. 3 (a). Since $H_{1}=C_{6}\left(d_{1}, b_{1}, d_{2}, b_{2}, d_{3}, b_{3}\right)$ is one of the two components of $\Gamma_{34},(\Gamma, \gamma)$ yields $\left\langle x_{1} \mid x_{1}^{3}\right\rangle \cong \mathbb{Z}_{3}$. So, $(\Gamma, \gamma)$ is a crystallization of $L(3,1)$. This completes the proof.

Lemma 21. There exists a unique 16-vertex 4 -colored graph $(\Gamma, \gamma)$ which is a crystallization of a closed connected 3-manifold whose fundamental group is $\mathbb{Z}_{5}$.

Proof. Let $(\Gamma, \gamma)$ be a 16-vertex crystallization of a connected closed 3-manifold $M$ and $\pi(M, *)=\mathbb{Z}_{5}$. Then $M$ can not have a non-trivial 2-fold cover and hence $M$ is orientable. Also, by Lemma $15, \psi(M)=16$ and hence, by Theorem $4,(\Gamma, \gamma)$ is the crystallization of $M$ with minimum number of vertices. Then, by Lemma $18,(\Gamma, \gamma)$ has no 2 -cycle. So, $\Gamma$ is a simple graph. Since $M$ is orientable, $\Gamma$ is bipartite. By Proposition 14 and Remark 16, $(\Gamma, \gamma)$ yields a presentation $\langle S \mid R\rangle$ of $\mathbb{Z}_{5}$ with $\varphi(S, R)=16$.

Claim 1. If $\left\langle S=\left\{x_{1}, x_{2}\right\} \mid R=\left\{r_{1}, r_{2}\right\}\right\rangle \in \mathcal{P}_{2}\left(\mathbb{Z}_{5}\right), \varphi(S, R)=16$ and $r_{3} \in \bar{R}$ is of minimum weight then $\left\{r_{1}, r_{2}, r_{3}\right\}=\left\{\left(x_{1}^{3} x_{2}^{-1}\right)^{ \pm 1},\left(x_{2}^{2} x_{1}^{-1}\right)^{ \pm 1},\left(x_{1}^{2} x_{2}\right)^{ \pm 1}\right\}$ or $\left\{\left(x_{1}^{2} x_{2}^{-1} x_{1} x_{2}^{-1}\right)^{ \pm 1},\left(x_{1} x_{2}\right)^{ \pm 1}\right.$, $\left.\left(x_{2}^{2} x_{1}^{-1} x_{2} x_{1}^{-1}\right)^{ \pm 1}\right\}$. (So, the set $\left\{r_{1}, r_{2}, r_{3}\right\}$ has 16 choices.) 

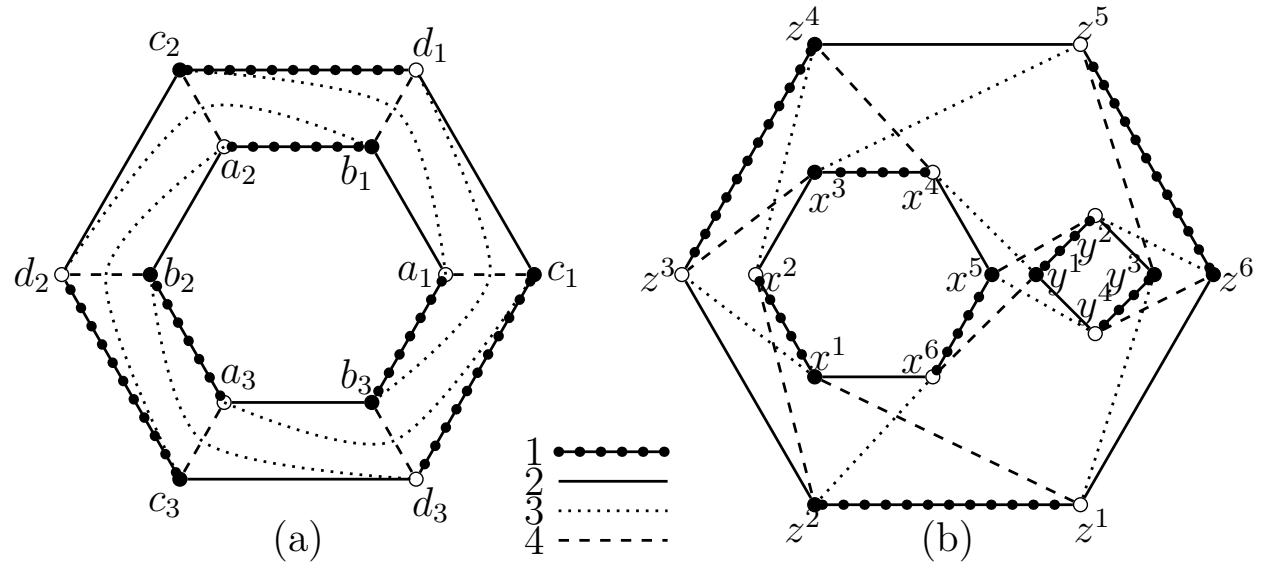

Figure 3: Crystallizations $\mathcal{K}_{3,1}$ and $\mathcal{M}_{2,3}$

Let $B$ be the set as in the proof of Lemma 15. Then $w \in F(S)$ and $4 \leqslant \lambda(w) \leqslant 6$ imply $w$ is dependent with an element of $B$. Since $\Gamma$ has no 2-cycle, $R$ has no element of weight less than 4 . Since $\varphi(S, R)=16$, we can assume that $4 \leqslant \lambda\left(r_{1}\right), \lambda\left(r_{2}\right) \leqslant 6$. Since $\langle S \mid R\rangle \in$ $\mathcal{P}_{2}\left(\mathbb{Z}_{5}\right) \backslash \mathcal{P}_{1}\left(\mathbb{Z}_{5}\right)$, the only possible choices of $\left\{r_{1}^{ \pm 1}, r_{2}^{ \pm 1}\right\}$ are $\left\{x_{1}^{3} x_{2}^{-1}, x_{2}^{2} x_{1}^{-1}\right\},\left\{x_{2}^{2} x_{1}^{-1}, x_{1}^{2} x_{2}\right\}$, $\left\{x_{1}^{3} x_{2}^{-1}, x_{1}^{2} x_{2}\right\},\left\{x_{1}^{2} x_{2}^{-1} x_{1} x_{2}^{-1}, x_{1} x_{2}\right\}$ or $\left\{x_{1}^{2} x_{2}^{-1} x_{1} x_{2}^{-1}, x_{2}^{2} x_{1}^{-1} x_{2} x_{1}^{-1}\right\}$. So, if $\langle S \mid R\rangle \in \mathcal{P}_{2}\left(\mathbb{Z}_{5}\right)$ and $\varphi(S, R)=16$, then $\left(r_{1}^{ \pm 1}, r_{2}^{ \pm 1}, r_{3}^{ \pm 1}\right)=\left(x_{1}^{3} x_{2}^{-1}, x_{2}^{2} x_{1}^{-1}, x_{1}^{2} x_{2}\right)$ or $\left(x_{1}^{2} x_{2}^{-1} x_{1} x_{2}^{-1}, x_{1} x_{2}\right.$, $\left.x_{2}^{2} x_{1}^{-1} x_{2} x_{1}^{-1}\right)$. This proves Claim 1 .

If $g_{i j}=2$ for some $i \neq j$ then $(\Gamma, \gamma)$ yields a presentation $\langle S \mid R\rangle \in \mathcal{P}_{1}\left(\mathbb{Z}_{5}\right)$ such that $\varphi(S, R)=16$ (see Remark 16), which is not possible by Eq. (3.1). Thus, $g_{i j} \geqslant 3$. Since (by Proposition 13) $g_{12}+g_{13}+g_{14}=16 / 2+2=10$, we can assume that $g_{12}=3=$ $g_{13}, g_{14}=4$. In particular, if we choose generators (resp., relations) corresponding to the components of $\Gamma_{12}\left(\operatorname{resp} ., \Gamma_{34}\right)$ then $(\Gamma, \gamma)$ yields a presentation $\langle S \mid R\rangle \in \mathcal{P}_{2}\left(\mathbb{Z}_{5}\right) \backslash \mathcal{P}_{1}\left(\mathbb{Z}_{5}\right)$ with $\varphi(S, R)=16$.

Claim 2. If $x_{1}, x_{2}$ are generators corresponding to two components of $\Gamma_{12}$ then the relations corresponding to the components of $\Gamma_{34}$ are $\left(x_{1}^{3} x_{2}^{-1}\right)^{\varepsilon_{1}}, x_{2}^{2} x_{1}^{-1},\left(x_{1}^{2} x_{2}\right)^{\varepsilon_{2}}$ for some $\varepsilon_{1}, \varepsilon_{2} \in$ $\{1,-1\}$.

Let $S, R, r_{1}, r_{2}, r_{3}$ be as in Claim 1 . Then by choosing $(i, j)=(3,4)$ or $(4,3)$ as in Eq. (2.6), by Claim 1, we can assume $\left(r_{1}, r_{2}, r_{3}\right)=\left(\left(x_{1}^{3} x_{2}^{-1}\right)^{ \pm 1}, x_{2}^{2} x_{1}^{-1},\left(x_{1}^{2} x_{2}\right)^{ \pm 1}\right)$ or $\left(\left(x_{1}^{2} x_{2}^{-1} x_{1} x_{2}^{-1}\right)^{ \pm 1},\left(x_{1} x_{2}\right)^{-1},\left(x_{2}^{2} x_{1}^{-1} x_{2} x_{1}^{-1}\right)^{ \pm 1}\right)$. In the first case, Claim 2 trivially holds. In the second case, $\tilde{r}_{2}=\left(x_{1} x_{3}^{-1} x_{2} x_{3}^{-1}\right)^{-1}$, where $x_{3}$ corresponds to the third component of $\Gamma_{12}$ (see Eq. (2.6)). By deleting $x_{2}$ and renaming $x_{3}$ by $x_{2}$ in $\tilde{r}_{2}^{ \pm 1}$, we get the new relation $x_{2}^{2} x_{1}^{-1}$. Claim 2 now follows from Claim 1 .

To construct $\tilde{r}_{i}$ as in Eq. (2.6), we can choose, without loss, $(i, j)=(4,3)$. Since $g_{23}=g_{14}=4, \Gamma_{14}$ and $\Gamma_{23}$ are of the form $C_{4} \sqcup C_{4} \sqcup C_{4} \sqcup C_{4}$. Again, $g_{12}=g_{34}=g_{24}=$ $g_{13}=3$ implies $\Gamma_{13}, \Gamma_{24}, \Gamma_{12}$ and $\Gamma_{34}$ are of the form $C_{4} \sqcup C_{6} \sqcup C_{6}$. Let $G_{1}, G_{2}, G_{3}$ be the components of $\Gamma_{12}$ and $H_{1}, H_{2}, H_{3}$ be the components of $\Gamma_{34}$ such that $x_{1}, x_{2}, x_{3}$ represent the generators corresponding to $G_{1}, G_{2}, G_{3}$ respectively and $\left(x_{1}^{3} x_{2}^{-1}\right)^{\varepsilon_{1}}, x_{2}^{2} x_{1}^{-1},\left(x_{1}^{2} x_{2}\right)^{\varepsilon_{2}}$ represent the relations corresponding to $H_{1}, H_{2}, H_{3}$ respectively. 
Let $G_{1}=C_{6}\left(x^{1}, \ldots, x^{6}\right), G_{2}=C_{4}\left(y^{1}, \ldots, y^{4}\right)$ and $G_{3}=C_{6}\left(z^{1}, \ldots, z^{6}\right)$. Then to form the relations $\left(x_{1}^{3} x_{2}^{-1}\right)^{\varepsilon_{1}}, x_{2}^{2} x_{1}^{-1},\left(x_{1}^{2} x_{2}\right)^{\varepsilon_{2}}$, we need to add the following: (i) two edges of color 4 between $G_{1}$ and $G_{2}$, (ii) four edges of colors 4 between $G_{1}$ and $G_{3}$, (iii) two edges of color 4 between $G_{2}$ and $G_{3}$. These give all the 8 edges in $\gamma^{-1}(4)$. Therefore, we must have the following: (a) two 4-cycles between $G_{1}$ and $G_{3}$ in $\Gamma_{14}$, (b) one 4-cycle between $G_{1}$ and $G_{2}$ in $\Gamma_{14}$, (c) one 4-cycle between $G_{2}$ and $G_{3}$ in $\Gamma_{14}$. So, the 4-cycle in $\Gamma_{24}$ is in between $G_{1}$ and $G_{3}$. Thus, up to an isomorphism, $\gamma^{-1}(4)$ is unique. In particular, we can assume that $\Gamma_{124}$ is as in Fig. 3 (b). Now, $y^{2} x^{5}$ is an edge of color 4 between $G_{1}$ and $G_{2}$. Thus, $y^{1}$ (resp., $y^{2}$ ) is in $H_{1}$ or $H_{2}$. Assume, without loss, $y^{1} \in H_{1}$. Then $y^{2} \in H_{2}$. Since $\Gamma$ is bipartite and $H_{2}$ represents $x_{2} x_{3}^{-1} x_{2} x_{1}^{-1}$, taking $v_{1}=x^{5}$ as in Eq. (2.6), $H_{2}=C_{4}\left(x^{5}, y^{2}, z^{6}, y^{4}\right)$. Since $\Gamma_{23}=C_{4} \sqcup C_{4} \sqcup C_{4} \sqcup C_{4}$ and $\Gamma_{13}=C_{4} \sqcup C_{6} \sqcup C_{6}$, we have $x^{4} y^{1}, x^{3} z^{5}, x^{2} z^{4}, x^{1} z^{3}, y^{3} z^{1}, x^{6} z^{2} \in \gamma^{-1}(3)$. Then $(\Gamma, \gamma)=\mathcal{M}_{2,3}$ of Fig. 3 (b). This completes the proof.

Lemma 22. There exists a unique 18-vertex crystallization of $S^{3} / Q_{8}$.

Proof. Let $(\Gamma, \gamma)$ be an 18-vertex crystallization of $S^{3} / Q_{8}$. By Lemma 15 and Theorem $4,(\Gamma, \gamma)$ is the crystallization of $S^{3} / Q_{8}$ with minimum number of vertices. So, by Lemma 18, $(\Gamma, \gamma)$ has no 2-cycle. Thus, $\Gamma$ is a simple graph. Since $S^{3} / Q_{8}$ is orientable, $\Gamma$ is bipartite. By Proposition 14 and Remark 16, $(\Gamma, \gamma)$ yields a presentation $\langle S \mid R\rangle$ of $Q_{8}$ with $\varphi(S, R)=18$. Again, $(\Gamma, \gamma)$ has no 2-cycle implies $g_{i j} \leqslant 4$ for $i \neq j$. By Proposition 13, $g_{12}+g_{13}+g_{14}=18 / 2+2=11$. Assume, without loss, that $g_{12}=3$ and $g_{13}=g_{14}=4$. Therefore, if we choose generators (resp., relations) correspond to the components of $\Gamma_{12}$ (resp., $\left.\Gamma_{34}\right)$ then $(\Gamma, \gamma)$ yields a presentation $\langle S \mid R\rangle \in \mathcal{P}_{2}\left(Q_{8}\right) \backslash \mathcal{P}_{1}\left(Q_{8}\right)$ with $\varphi(S, R)=18$. Then by the proof of part (v) in Lemma $15, R=\left\{\left(x_{2}^{2} x_{1}^{-2}\right)^{\varepsilon_{1}},\left(x_{1} x_{2} x_{1} x_{2}^{-1}\right)^{\varepsilon_{2}},\left(x_{2} x_{1} x_{2} x_{1}^{-1}\right)^{\varepsilon_{3}}\right\}$ for some $\varepsilon_{1}, \varepsilon_{2}, \varepsilon_{3} \in\{1,-1\}$. Then, by choosing $(i, j)=(3,4)$ or $(4,3)$ as in Eq. (2.6), we can assume that the three relations correspond to components of $\Gamma_{34}$ are $\left(x_{2}^{2} x_{1}^{-2}\right)^{\varepsilon_{1}},\left(x_{1} x_{2} x_{1} x_{2}^{-1}\right)^{\varepsilon_{2}}, x_{2} x_{1} x_{2} x_{1}^{-1}$, for some $\varepsilon_{1}, \varepsilon_{2} \in\{-1,1\}$. Since $\Gamma$ has no 2-cycle, $\Gamma_{i j}=C_{4} \sqcup C_{4} \sqcup C_{4} \sqcup C_{6}$ for $1 \leqslant i \leqslant 2$ and $3 \leqslant j \leqslant 4$. Let $G_{1}, G_{2}, G_{3}$ be the components of $\Gamma_{12}$ and $H_{1}, H_{2}, H_{3}$ be the components of $\Gamma_{34}$ such that $x_{1}, x_{2}, x_{3}$ represent the generators corresponding to $G_{1}, G_{2}, G_{3}$ and $\left(x_{2}^{2} x_{1}^{-2}\right)^{\varepsilon_{1}}, x_{2} x_{1} x_{2} x_{1}^{-1},\left(x_{1} x_{2} x_{1} x_{2}^{-1}\right)^{\varepsilon_{2}}$ represent the relations corresponding to $H_{1}, H_{2}, H_{3}$ respectively. Then $G_{i}, H_{i}$ are 6 -cycles for $1 \leqslant i \leqslant 3$. Let $G_{1}=C_{6}\left(a_{1}, \ldots, a_{6}\right), G_{2}=$ $C_{6}\left(b_{1}, \ldots, b_{6}\right), G_{3}=C_{6}\left(c_{1}, \ldots, c_{6}\right)$. Again, to form these relations, there are exactly three edges with color 4 between $G_{i}$ and $G_{j}$ for $i \neq j$. Since each of $\Gamma_{14}$ and $\Gamma_{24}$ has three 4-cycles, the three edges with color 4 between $G_{i}$ and $G_{j}$ for $i \neq j$, yield two 4-cycles. Then, up to an isomorphism, $\Gamma_{124}$ is as in Fig. 4. Same arguments hold for color 3.

To construct $\tilde{r}_{k}$ as in Eq. (2.6), choose $(i, j)=(4,3)$. Since $H_{2}$ presents the relation $x_{2} x_{1} x_{2} x_{1}^{-1}$, up to isomorphism, the starting vertex $v_{1}$ (as in Eq. (2.6)) is $a_{2}$ or $a_{3}$. If $v_{1}=a_{2}$ then $H_{2}=C_{6}\left(a_{2}, b_{3}, c_{4}, a_{5}, c_{2}, b_{5}\right)$ or $C_{6}\left(a_{2}, b_{3}, c_{4}, a_{5}, c_{6}, b_{1}\right)$. In the first case, if $b_{4} c_{3} \in \gamma^{-1}(3)$, then $b_{4} c_{3}$ lies in a cycle of size at least 8 in $\Gamma_{23}$, which is not possible. Then the 4-cycle in $\Gamma_{13}$ between $G_{2}$ and $G_{3}$ must be $C_{4}\left(b_{1}, b_{2}, c_{5}, c_{6}\right)$. But this is not possible since $b_{1} c_{6} \in \gamma^{-1}(4)$. In the second case, if $b_{2} c_{5} \in \gamma^{-1}(3)$, then $b_{2} c_{5}$ lies in a cycle of size at least 8 in $\Gamma_{13}$, which is not possible. Then the 4-cycle in $\Gamma_{23}$ between $G_{2}$ and $G_{3}$ must be 


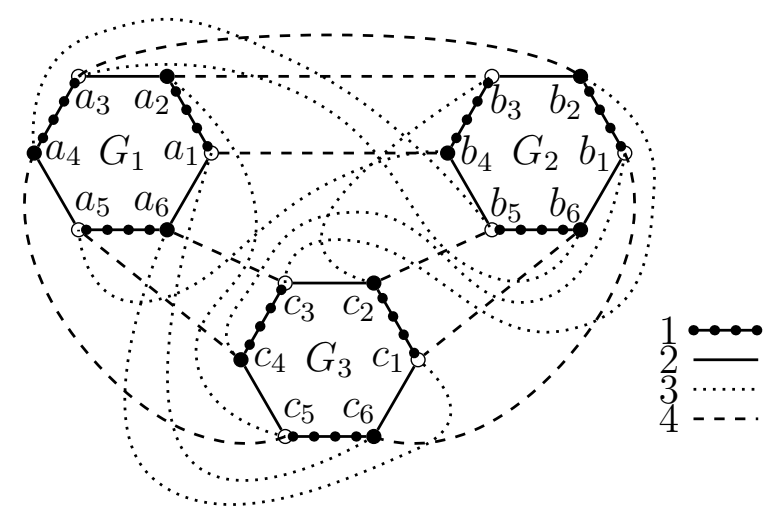

Figure 4: Crystallization $\mathcal{J}_{3}$ of $S^{3} / \mathbb{Q}_{8}$

$C_{4}\left(b_{4}, b_{5}, c_{2}, c_{3}\right)$. Again, this is not possible since $b_{5} c_{2} \in \gamma^{-1}(4)$. Thus, $v_{1}=a_{3}$. Now, if $b_{2} c_{5}$ is an edge of color 3 then $a_{4} c_{1}$ and $a_{3} b_{6}$ must be edges of color 3 . Then $b_{5} c_{2}$ must be an edge of color 3 to make a 6 -cycle in $\Gamma_{13}$, which is a contradiction (since $b_{5} c_{2}$ is already an edge of color 4$)$. Thus, $H_{2}=C_{6}\left(a_{3}, b_{2}, c_{3}, a_{6}, c_{1}, b_{6}\right)$. Since the three edges with color 3 between $G_{2}$ and $G_{3}$ yield two 4-cycles (in $\Gamma_{13}$ and $\Gamma_{23}$ ), $b_{1} c_{4}, b_{3} c_{2}$ must be edges of color 3 between $G_{2}$ and $G_{3}$. To make a 6 -cycle in $\Gamma_{13}, a_{5} b_{4}$ must be an edge of color 3 . By similar arguments, $a_{1} c_{6}, a_{2} c_{5}, a_{4} b_{5} \in \gamma^{-1}(3)$. Then, $(\Gamma, \gamma)=\mathcal{J}_{3}$ of Fig. 4.

Now, the components $H_{1}=C_{6}\left(a_{2}, b_{3}, c_{2}, b_{5}, a_{4}, c_{5}\right)$ and $H_{3}=C_{6}\left(b_{4}, a_{1}, c_{6}, b_{1}, c_{4}, a_{5}\right)$ of $\Gamma_{34}$ yield the relations $x_{2}^{2} x_{1}^{-2}$ and $x_{1} x_{2} x_{1} x_{2}^{-1}$ respectively. Thus $(\Gamma, \gamma)$ yields the presentation $\left\langle x_{1}, x_{2} \mid x_{2}^{2} x_{1}^{-2}, x_{1} x_{2} x_{1} x_{2}^{-1}\right\rangle \cong Q_{8}$. This completes the proof.

Lemma 23. There exists a unique 24-vertex crystallization of $S^{1} \times S^{1} \times S^{1}$.

Proof. Let $(\Gamma, \gamma)$ be a 24-vertex crystallization of $\left(S^{1}\right)^{3}$. By Lemma 15 and Theorem 4, $(\Gamma, \gamma)$ is the crystallization of $\left(S^{1}\right)^{3}$ with minimum number of vertices. So, by Lemma 18, $(\Gamma, \gamma)$ has no 2-cycle. Thus, $\Gamma$ is a simple graph. Since $\left(S^{1}\right)^{3}$ is orientable, $\Gamma$ is bipartite. By Proposition 14 and Remark 16, $(\Gamma, \gamma)$ yields a presentation $\langle S \mid R\rangle$ of $\mathbb{Z}^{3}$ with $\varphi(S, R)=24$. Since any presentation of $\mathbb{Z}^{3}$ has at least three generators, $g_{i j} \geqslant 4$ for $i \neq j$. By Proposition 13, $g_{12}+g_{13}+g_{14}=14$ and $g_{i j}=g_{k l}$ for $i, j, k, l$ distinct.

Claim. $(\Gamma, \gamma)$ does not yield a presentation $\langle S \mid R\rangle \in \mathcal{P}_{4}\left(\mathbb{Z}^{3}\right) \backslash \mathcal{P}_{3}\left(\mathbb{Z}^{3}\right)$ with $\varphi(S, R)=24$.

Assume $\langle S \mid R\rangle \in \mathcal{P}_{4}\left(\mathbb{Z}^{3}\right) \backslash \mathcal{P}_{3}\left(\mathbb{Z}^{3}\right)$, where $S=\left\{x_{1}, x_{2}, x_{3}, x_{4}\right\}$. Then $A:=\left\{\left(x_{k}^{2} x_{l}^{-1}\right)^{ \pm 1}\right.$, $\left(x_{j} x_{k}^{-1} x_{j} x_{l}^{-1}\right)^{ \pm 1}, x_{i} x_{j}^{-1} x_{k} x_{l}^{-1},\left(x_{j} x_{k} x_{l}^{-1}\right)^{ \pm 1},\left(x_{k} x_{l}^{-1}\right)^{2}, x_{l}^{ \pm 2}: i, j, k, l$ are distinct $\}$ is the set of all relations of weight four. Since $\Gamma$ has no 2-cycle, $R$ has no element of weight two. This implies that $R$ has at least three elements of weights four. Since $\mathbb{Z}^{3}$ has no torsion element, $x_{l}^{ \pm 2},\left(x_{k} x_{l}^{-1}\right)^{2} \notin R$. Consider an element $w \in R \cap A$. Assume, without loss, $w=\left(w_{1} x_{4}^{-1}\right)^{ \pm 1}$. Then $\left\langle S_{1} \mid R_{1}\right\rangle \in \mathcal{P}_{3}\left(\mathbb{Z}^{3}\right)$, where $S_{1}=\left\{x_{1}, x_{2}, x_{3}\right\}$ and $R_{1}$ consists of the elements $\bar{r}$, where $\bar{r}$ can be obtained from a relation $r \in R$ by replacing $x_{4}$ by $w_{1}$. Let $A(w):=\left\{\bar{r}: r \in A \backslash\left\{w^{ \pm 1}\right\}\right\}$. Observe that the weights of the elements in $A(w)$ are 6 or 8.

Since $\left\langle S_{1} \mid R_{1}\right\rangle \in \mathcal{P}_{3}\left(\mathbb{Z}^{3}\right) \backslash \mathcal{P}_{2}\left(\mathbb{Z}^{3}\right)$, we have $N\left(R_{1}\right)=N\left(R_{0}\right)$, where $R_{0}=\left\{x_{1} x_{2} x_{1}^{-1} x_{2}^{-1}\right.$, $\left.x_{1} x_{3} x_{1}^{-1} x_{3}^{-1}, x_{2} x_{3} x_{2}^{-1} x_{3}^{-1}\right\}$ and hence $N\left(R_{1}\right)$ has no element of weight less than 6 (see 
the proof of part (v) of Lemma 15). Again, since $\#\left(R_{1} \cap A(w)\right) \geqslant 2, R_{1}$ has at least two elements of weights 6 or 8 . Observe that $D:=\left\{x_{i} x_{j} x_{i}^{-1} x_{j}^{-1}, x_{i} x_{j}^{-1} x_{k} x_{i}^{-1} x_{j} x_{k}^{-1}\right.$, $\left.x_{i} x_{j} x_{k}^{-1} x_{i}^{-1} x_{k} x_{j}^{-1}, x_{i} x_{j} x_{i}^{-1} x_{k} x_{j}^{-1} x_{k}^{-1}:\{i, j, k\}=\{1,2,3\}\right\}$ is the set of all relations of weights at most 8 in $N\left(R_{0}\right)$. So, $R_{1}$ has at least two independent relations from $D \cap A(w)$. But $D \cap A(w)$ does not contain two such elements, a contradiction. This proves the claim.

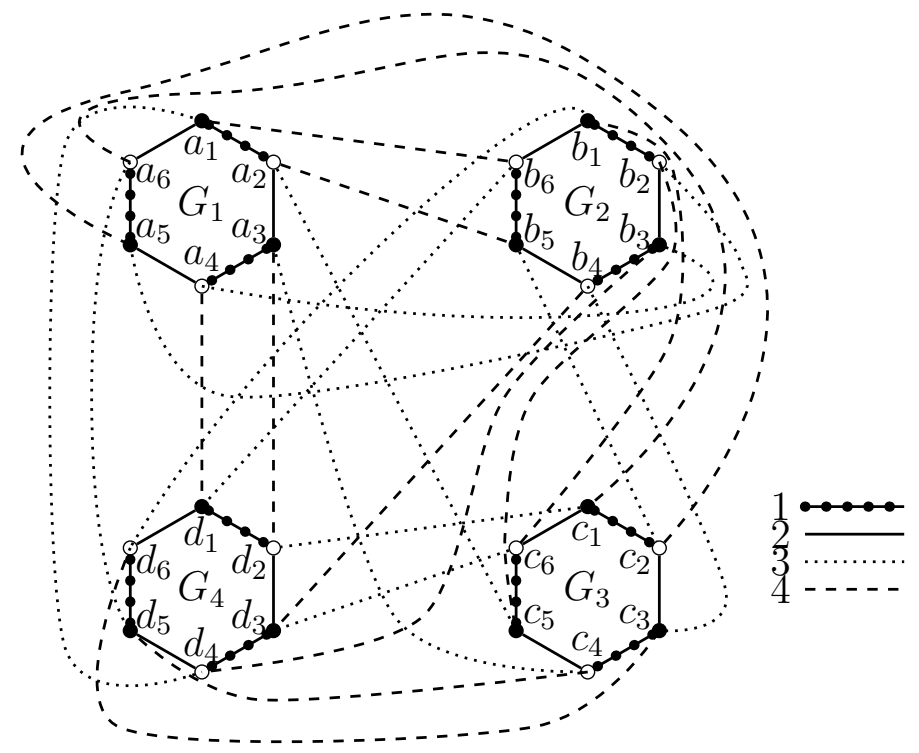

Figure 5: Crystallization $\mathcal{J}_{4}$ of $S^{1} \times S^{1} \times S^{1}$

By the claim, $g_{i j} \neq 5$ for all $1 \leqslant i \neq j \leqslant 4$. So, we can assume that $g_{12}=g_{13}=4$ and $g_{14}=6$. Then all the components of $\Gamma_{14}$ and $\Gamma_{23}$ are 4-cycles. Let $\Gamma_{12}=G_{1} \sqcup \cdots \sqcup G_{4}$ and $\Gamma_{34}=H_{1} \sqcup \cdots \sqcup H_{4}$ such that $x_{1}, \ldots, x_{4}$ represent the generators corresponding to $G_{1}, \ldots, G_{4}$ respectively and $r_{1}, \ldots, r_{4}$ represent the relations corresponding to $H_{1}, \ldots, H_{4}$ respectively. To construct $\tilde{r}_{k}$ as in Eq. (2.6), choose $(i, j)=(4,3)$. Thus $(\Gamma, \gamma)$ yields a presentation $\left\langle S=\left\{x_{1}, x_{2}, x_{3}\right\} \mid R=\left\{r_{1}, r_{2}, r_{3}\right\}\right\rangle \in \mathcal{P}_{3}\left(\mathbb{Z}^{3}\right) \backslash \mathcal{P}_{2}\left(\mathbb{Z}^{3}\right)$ with $\varphi(S, R)=24$. Then $R$ contains three independent relations of weight 6 from the set $\left\{x_{i} x_{j} x_{i}^{-1} x_{j}^{-1}, x_{i} x_{j}^{-1} x_{k} x_{i}^{-1} x_{j} x_{k}^{-1}:\{i, j, k\}=\{1,2,3\}\right\}$ (see the proof of part (v) of Lemma 15). Without loss of generality, we can assume that $R=\left\{x_{1} x_{2} x_{1}^{-1} x_{2}^{-1},\left(x_{2} x_{3} x_{2}^{-1} x_{3}^{-1}\right)^{\varepsilon_{1}}\right.$, $\left.\left(x_{1} x_{3} x_{1}^{-1} x_{3}^{-1}\right)^{\varepsilon_{2}}\right\}$ for some $\varepsilon_{1}, \varepsilon_{2} \in\{1,-1\}$. Then, all the components of $\Gamma_{12}$ and $\Gamma_{34}$ are 6cycles. Similarly, all the components of $\Gamma_{13}$ and $\Gamma_{24}$ are 6 -cycles. Let $G_{1}=C_{6}\left(a_{1}, \ldots, a_{6}\right)$, $G_{2}=C_{6}\left(b_{1}, \ldots, b_{6}\right), G_{3}=C_{6}\left(c_{1}, \ldots, c_{6}\right)$ and $G_{4}=C_{6}\left(d_{1}, \ldots, d_{6}\right)$. To form the relations, there are exactly two edges of color 3 (resp., 4 ) between $G_{i}$ and $G_{j}$ for $1 \leqslant i \neq j \leqslant 4$. Then, up to an isomorphism, $\Gamma_{124}$ is as in Fig. 5 . Now for the relation $x_{1} x_{2} x_{1}^{-1} x_{2}^{-1}$, we can choose $v_{1}=b_{6}$ as in Eq. (2.6). Then the cycle for $x_{1} x_{2} x_{1}^{-1} x_{2}^{-1}$ is $H_{1}=C_{6}\left(b_{6}, a_{1}, d_{4}, b_{3}, a_{4}, d_{1}\right)$. Since $\Gamma_{23}$ consists of 4 -cycles, it follows that $a_{6} d_{5}, a_{5} b_{2}, b_{1} d_{6} \in \gamma^{-1}(3)$. Then the cycle for the relation $x_{2} x_{3} x_{2}^{-1} x_{3}^{-1}$ is $H_{2}=C_{6}\left(c_{6}, b_{1}, d_{6}, c_{3}, b_{4}, d_{3}\right)$. Again (since $\Gamma_{23}$ is union of 4 -cycles and $\Gamma_{13}$ is union of 6-cycles), $d_{2} c_{1}, b_{5} c_{2}, a_{3} c_{4}, a_{2} c_{5} \in \gamma^{-1}(3)$. Then $(\Gamma, \gamma)=\mathcal{J}_{4}$ of Fig. 5 . 
Now, the components $H_{1}, H_{2}$ and $H_{3}=C_{6}\left(c_{1}, a_{6}, d_{5}, c_{4}, a_{3}, d_{2}\right)$ yield the relations $x_{1} x_{2} x_{1}^{-1} x_{2}^{-1}, x_{2} x_{3} x_{2}^{-1} x_{3}^{-1}$ and $x_{1} x_{3} x_{1}^{-1} x_{3}^{-1}$ respectively. Thus $(\Gamma, \gamma)$ yields the presentation $\left\langle x_{1}, x_{2}, x_{3} \mid x_{1} x_{2} x_{1}^{-1} x_{2}^{-1}, x_{2} x_{3} x_{2}^{-1} x_{3}^{-1}, x_{1} x_{3} x_{1}^{-1} x_{3}^{-1}\right\rangle \cong \mathbb{Z}^{3}$. This completes the proof.

Remark 24. The crystallizations $\mathcal{K}_{2,1}, \mathcal{K}_{3,1}$ and $\mathcal{M}_{3,2}$ (in Figures 2 and 3) were originally found by Gagliardi et al. $([8,10])$. The first two have the following natural generalization: Consider the bipartite graph $\Gamma$ consists of two disjoint $2 p$-cycles $G_{1}=$ $C_{2 p}\left(a_{1}, b_{1}, \ldots, a_{p}, b_{p}\right), G_{2}=C_{2 p}\left(c_{1}, d_{1}, \ldots, c_{p}, d_{p}\right)$ together with $4 p$ edges $a_{i} c_{i}, b_{i} d_{i}, a_{i} c_{i+q}$, $b_{i} d_{i+q}$ for $1 \leqslant i \leqslant p$. Consider the edge-coloring $\gamma$ with colors $1,2,3,4$ of $\Gamma$ as: $\gamma\left(b_{i} a_{i+1}\right)=$ $\gamma\left(d_{i} c_{i+1}\right)=1, \gamma\left(a_{i} b_{i}\right)=\gamma\left(c_{i} d_{i}\right)=2, \gamma\left(a_{i} c_{i+q}\right)=\gamma\left(b_{i} d_{i+q}\right)=3$ and $\gamma\left(a_{i} c_{i}\right)=\gamma\left(b_{i} d_{i}\right)=4$, $1 \leqslant i \leqslant p$. (Summations in the subscripts are modulo $p$.) Then, $\mathcal{K}_{p, q}=(\Gamma, \gamma)$ is a $4 p$ vertex crystallization of $L(p, q)$, for $p \geqslant 2$ and $q \geqslant 1$. This series is more or less known in the literature. In the next section, we present some generalizations of $\mathcal{M}_{3,2}$.

\section{Two series of crystallizations of lens spaces}

Generalizing the construction of $\mathcal{M}_{3,2}$ (Fig. 3 (b)) we have constructed the following series of crystallizations.

\subsection{A $4(k+q-1)$-vertex crystallization of $L(k q-1, q)$}

Let $q \geqslant 3$. For each $k \geqslant 2$, we construct a $4(k+q-1)$-vertex 4-colored simple graph $\mathcal{M}_{k, q}=\left(\Gamma^{k}, \gamma^{k}\right)$ with the color set $\{1,2,3,4\}$ inductively which yields the presentation $\left\langle x, y \mid x^{q} y^{-1}, y^{k} x^{-1}\right\rangle$. For this, we want $g_{12}^{k}=g_{34}^{k}=3$. Then, without loss, $g_{13}^{k}=g_{24}^{k}=$ $k+q-2$ and $g_{14}^{k}=g_{23}^{k}=k+q-1$, where $g_{i j}^{k}$ is the number of components of $\Gamma_{i j}^{k}$ for $i \neq j$. These imply, $\Gamma_{14}^{k}$ and $\Gamma_{23}^{k}$ must be union of 4-cycles and $\Gamma_{13}^{k}$ (resp., $\Gamma_{24}^{k}$ ) has two 6-cycles and $(k+q-4)$ 4-cycles. Then, by Proposition $13, \mathcal{M}_{k, q}$ would be a crystallization of some connected closed 3-manifold $M_{k}$.

$\boldsymbol{k}=\mathbf{2}$ case: The crystallization $\mathcal{M}_{2, q}$ is given in Fig. 6 . Then, the components of $\Gamma_{12}^{2}$ are $G_{1}=C_{2 q}\left(x^{1}, \ldots, x^{2 q}\right), G_{2}=C_{4}\left(y^{1}, \ldots, y^{4}\right), G_{3}=C_{2 q}\left(z^{1}, \ldots, z^{2 q}\right)$ and the components of $\Gamma_{34}^{2}$ are $H_{1}=C_{2 q}\left(y^{1}, x^{2 q}, z^{2}, x^{2}, \ldots, z^{2 q-2}, x^{2 q-2}\right), H_{2}=C_{4}\left(x^{2 q-1}, y^{2}, z^{2 q}, y^{4}\right), H_{3}=$ $C_{2 q}\left(z^{2 q-1}, y^{3}, z^{1}, x^{1}, \ldots, z^{2 q-3}, x^{2 q-3}\right)$. Let $x, y$ be the generators corresponding to $G_{1}$ and $G_{2}$ respectively. To construct $\tilde{r}_{1}$ (resp., $\tilde{r}_{2}$ ) as in Eq. $(2.6)$, choose $(i, j)=(4,3)$ and $v_{1}=y^{1}$ (resp., $\left.v_{1}=x^{2 q-1}\right)$. Then $H_{1}$ and $H_{2}$ represent the relations $x^{q} y^{-1}$ and $y^{2} x^{-1}$ respectively. Therefore, by Proposition $14, \pi\left(M_{2}, *\right) \cong\left\langle x, y \mid x^{q} y^{-1}, y^{2} x^{-1}\right\rangle \cong \mathbb{Z}_{2 q-1}$.

Let $T$ and $T_{2}$ be the 3-dimensional simplicial cell complexes represented by the color graphs $\left.\Gamma^{2}\right|_{\left\{x^{1}, x^{2}, x^{3}, z^{3}\right\}}$ and $\left.\Gamma^{2}\right|_{V\left(\Gamma^{2}\right) \backslash\left\{x^{1}, x^{2}, x^{3}, z^{3}\right\}}$ respectively. Then $|T|$ and $\left|T_{2}\right|$ are solid tori and the facets (2-cells) of $T \cap T_{2}$ are $x_{2}^{1}, x_{4}^{1}, x_{3}^{2}, x_{4}^{2}, x_{1}^{3}, x_{3}^{3}, z_{1}^{3}, z_{2}^{3}$. Thus, $\left|T \cap T_{2}\right|$ is a torus (see Fig. $7(\mathrm{~b})$ ) with $\pi_{1}\left(\left|T \cap T_{2}\right|, v_{1}\right)=\left\langle\alpha=[a], \beta=[b] \mid \alpha \beta \alpha^{-1} \beta^{-1}\right\rangle$, where $a=x_{34}^{2} x_{34}^{3}$ and $b=x_{34}^{3} z_{13}^{3} z_{23}^{3}$. Then $b=x_{34}^{1} x_{13}^{1} x_{23}^{1}=\partial\left(x_{3}^{1}\right) \sim 1$ in $|T|$. Therefore, $\pi_{1}\left(|T|, v_{1}\right)=\langle\alpha, \beta \mid \beta\rangle$.

Since $\alpha \beta=\beta \alpha$ in $|T| \cap\left|T_{2}\right|, a b \sim b a$ in $\left|T_{2}\right|$. Now $a b=\left(x_{34}^{2} x_{34}^{1}\right)\left(x_{34}^{1} x_{13}^{1} x_{23}^{1}\right) \sim$ $x_{34}^{2} x_{13}^{1} x_{23}^{1}=x_{34}^{2} x_{13}^{2} x_{23}^{1} \sim x_{23}^{2} x_{23}^{1}$. Therefore, $a^{2} b \sim a b a \sim x_{23}^{2} x_{23}^{1} x_{34}^{2} x_{34}^{1}=x_{23}^{2} x_{23}^{2 q} x_{34}^{2 q} x_{34}^{1} \sim$ $x_{23}^{2} x_{13}^{2 q} x_{34}^{1}=x_{23}^{2} z_{13}^{1} z_{34}^{1} \sim x_{23}^{2} z_{23}^{1}$. Thus, $a^{3} b \sim x_{34}^{2} x_{34}^{1} x_{23}^{2} z_{23}^{1}=x_{34}^{2} x_{34}^{3} x_{23}^{3} z_{23}^{1} \sim x_{34}^{2} x_{13}^{3} z_{23}^{1}=$ 


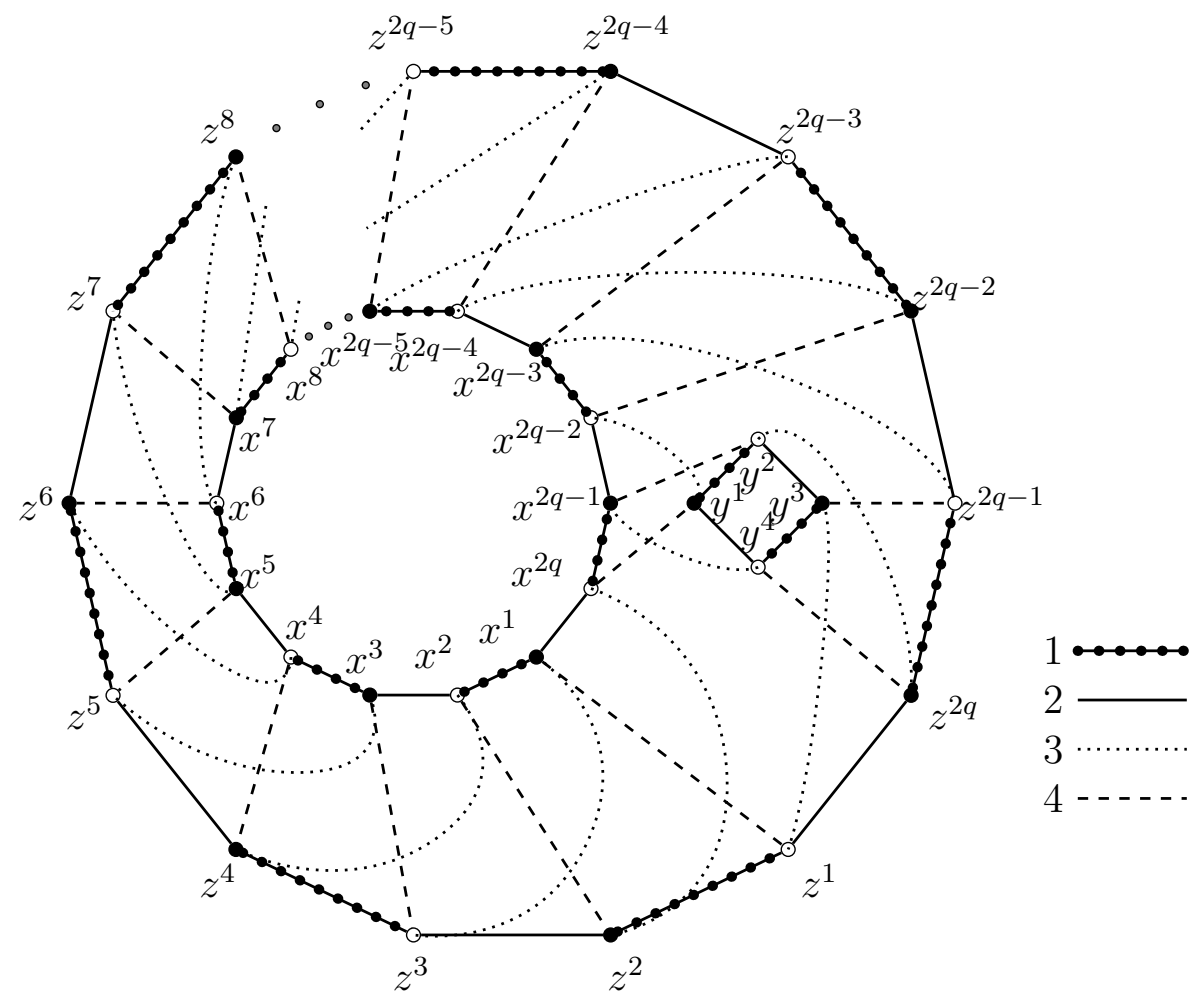

Figure 6: Crystallization $\mathcal{M}_{2, q}$ of $\mathrm{L}(2 q-1, q)$

$x_{34}^{4} x_{13}^{4} z_{23}^{1} \sim x_{23}^{4} z_{23}^{1}$. Similarly, $a^{q-1} b \sim x_{23}^{2 q-4} z_{23}^{1}$. Therefore, $a^{q} b \sim x_{34}^{2} x_{34}^{1} x_{23}^{2 q-4} z_{23}^{1}=$ $x_{34}^{2} x_{34}^{2 q-3} x_{23}^{2 q-3} z_{23}^{1} \sim x_{34}^{2} x_{13}^{2 q-3} z_{23}^{1}=x_{34}^{2 q-2} x_{13}^{2 q-2} z_{23}^{1} \sim x_{23}^{2 q-2} z_{23}^{1}=x_{23}^{2 q-1} z_{23}^{1} \sim x_{34}^{2 q-1} x_{13}^{2 q-1} z_{23}^{1}=$ $x_{34}^{2 q-1} z_{13}^{1} z_{23}^{1} \sim x_{34}^{2 q-1} z_{34}^{1}=x_{34}^{2 q-1} z_{34}^{2 q-1}$. Since $k=2$, we have $z_{13}^{2 q-1}=z_{13}^{2 q}$. This implies, $a^{2 q-1} b^{2} \sim x_{34}^{2 q-1} z_{34}^{2 q-1} x_{23}^{2 q-4} z_{23}^{1}=x_{34}^{2 q-1} z_{34}^{2 q-1} z_{23}^{2 q-1} z_{23}^{1} \sim x_{34}^{2 q-1} z_{13}^{2 q-1} z_{23}^{1}=z_{34}^{2 q} z_{13}^{2 q} z_{23}^{2 q}=\partial\left(z_{3}^{2 q}\right) \sim$ 1 in $\left|T_{2}\right|$. Thus $\pi_{1}\left(\left|T_{2}\right|, v_{1}\right)=\left\langle\alpha, \beta \mid \alpha^{2 q-1} \beta^{2}, \alpha \beta \alpha^{-1} \beta^{-1}\right\rangle$. This implies that (see the second paragraph of Subsection 2.3) $|T| \cup\left|T_{2}\right|=L(2 q-1,2)$. Therefore, $\mathcal{M}_{2, q}$ is a crystallization of $L(2 q-1,2) \cong L(2 q-1, q)$.

$\boldsymbol{k}=\mathbf{3}$ case: Here $z_{13}^{2 q-1} \neq z_{13}^{2 q}$. Let

$$
\begin{aligned}
\Gamma^{3}= & \left(V\left(\Gamma^{2}\right) \cup\left\{y^{5}, y^{6}, z^{2 q+1}, z^{2 q+2}\right\}, E\left(\Gamma^{2}\right) \backslash\left\{y^{2} z^{2 q}, y^{3} z^{1}, y^{3} y^{4}, z^{2 q-1} z^{2 q}\right\} \cup\left\{y^{3} y^{5}, y^{5} y^{6},\right.\right. \\
& \left.\left.y^{6} y^{4}, z^{2 q-1} z^{2 q+1}, z^{2 q+1} z^{2 q+2}, z^{2 q+2} z^{2 q}, y^{5} z^{2 q+1}, y^{6} z^{2 q+2}, y^{2} z^{2 q+1}, y^{3} z^{2 q+2}, y^{5} z^{2 q}, y^{6} z^{1}\right\}\right) .
\end{aligned}
$$

Consider the following coloring $\gamma^{3}$ on the edges of $\Gamma^{3}$ : same colors on the old edges as in $\mathcal{M}_{2, q}$, color 1 on the edges $y^{3} y^{5}, y^{6} y^{4}, z^{2 q-1} z^{2 q+1}, z^{2 q+2} z^{2 q}$, color 2 on the edges $y^{5} y^{6}$, $z^{2 q+1} z^{2 q+2}$, color 3 on the edges $y^{2} z^{2 q+1}, y^{3} z^{2 q+2}, y^{5} z^{2 q}, y^{6} z^{1}$ and color 4 on the edges $y^{5} z^{2 q+1}, y^{6} z^{2 q+2}$ (see Fig. $7(a)$ ). Let $T$ be as in the case $k=2$ and $T_{3}$ be the cell complex represented by the colored graph $\left.\Gamma^{3}\right|_{V\left(\Gamma^{3}\right) \backslash\left\{x^{1}, x^{2}, x^{3}, z^{3}\right\}}$.

Then, $a^{2 q-1} b^{2} \sim z_{34}^{2 q} z_{13}^{2 q-1} z_{23}^{2 q}=z_{34}^{2 q+1} z_{13}^{2 q+1} z_{23}^{2 q} \sim z_{23}^{2 q+1} z_{23}^{2 q}=z_{23}^{2 q+2} z_{23}^{2 q}$. This implies, $a^{3 q-1} b^{3}=\left(a^{q} b\right)\left(a^{2 q-1} b^{2}\right) \sim\left(x_{34}^{2 q-1} z_{34}^{2 q-1}\right)\left(z_{23}^{2 q+2} z_{23}^{2 q}\right)=z_{34}^{2 q} z_{34}^{2 q+2} z_{23}^{2 q+2} z_{23}^{2 q} \sim z_{34}^{2 q} z_{13}^{2 q+2} z_{23}^{2 q}=$ $z_{34}^{2 q} z_{13}^{2 q} z_{23}^{2 q}=\partial\left(z_{3}^{2 q}\right) \sim 1$ in $\left|T_{3}\right|$. Thus, $\pi_{1}\left(\left|T_{3}\right|, v_{1}\right)=\left\langle\alpha, \beta \mid \alpha^{3 q-1} \beta^{3}, \alpha \beta \alpha^{-1} \beta^{-1}\right\rangle$ and hence $|T| \cup\left|T_{3}\right|=L(3 q-1,3)$. Therefore, $\mathcal{M}_{3, q}$ is a crystallization of $L(3 q-1,3) \cong L(3 q-1, q)$. 


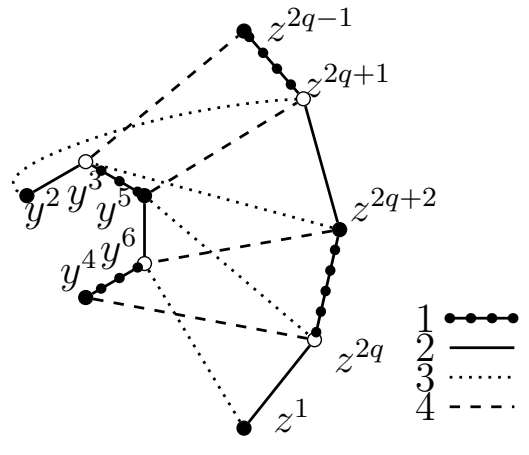

Figure 7: (a) Crystallization $\mathcal{M}_{3, q}$ of $\mathrm{L}(3 q-1, q)$

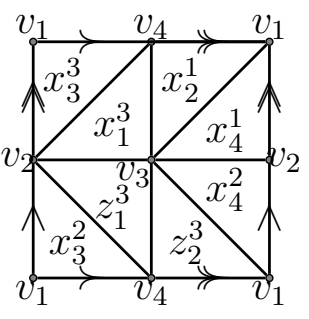

(b) $\left|T_{1} \cap T_{k}\right|$

$\boldsymbol{k} \geqslant 4$ case: Consider the graph

$$
\begin{aligned}
\Gamma^{k}= & \left(V\left(\Gamma^{k-1}\right) \cup\left\{y^{2 k-1}, y^{2 k}, z^{2 q+2 k-5}, z^{2 q+2 k-4}\right\}, E\left(\Gamma^{k-1}\right) \backslash\left\{y^{2 k-3} z^{2 q}, y^{2 k-2} z^{1}, y^{2 k-2} y^{4},\right.\right. \\
& \left.z^{2 q+2 k-6} z^{2 q}\right\} \cup\left\{y^{2 k-2} y^{2 k-1}, y^{2 k-1} y^{2 k}, y^{2 k} y^{4}, z^{2 q+2 k-6} z^{2 q+2 k-5}, z^{2 q+2 k-5} z^{2 q+2 k-4},\right. \\
& \left.\left.z^{2 q+2 k-4} z^{2 q}, y^{2 k-1} z^{2 q+2 k-5}, y^{2 k} z^{2 q+2 k-4}, y^{2 k-3} z^{2 q+2 k-5}, y^{2 k-2} z^{2 q+2 k-4}, y^{2 k-1} z^{2 q}, y^{2 k} z^{1}\right\}\right) .
\end{aligned}
$$

Also, consider the following coloring $\gamma^{k}$ on the edges of $\Gamma^{k}$ : same colors on the old edges as in $\mathcal{M}_{k-1, q}$, color 1 on the edges $y^{2 k-2} y^{2 k-1}, y^{2 k} y^{4}, z^{2 q+2 k-6} z^{2 q+2 k-5}, z^{2 q+2 k-4} z^{2 q}$, color 2 on the edges $y^{2 k-1} y^{2 k}, z^{2 q+2 k-5} z^{2 q+2 k-3}$, color 3 on the edges $y^{2 k-3} z^{2 q+2 k-5}, y^{2 k-2} z^{2 q+2 k-4}$, $y^{2 k-1} z^{2 q}, y^{2 k} z^{1}$ and color 4 on the edges $y^{2 k-1} z^{2 q+2 k-5}, y^{2 k} z^{2 q+2 k-4}$. Let $T$ be as in the case $k=2$ and $T_{k}$ be the cell complex represented by the colored graph $\left.\Gamma^{k}\right|_{V\left(\Gamma^{k}\right) \backslash\left\{x^{1}, x^{2}, x^{3}, z^{3}\right\}}$.

Claim. $a^{k q-1} b^{k} \sim z_{34}^{2 q} z_{13}^{2 q+2 k-4} z_{23}^{2 q}$ in $\left|T_{k}\right|$.

We prove the claim by induction. It is true for $k=3$. Assume that $a^{(k-1) q-1} b^{k-1} \sim$ $z_{34}^{2 q} z_{13}^{2 q+2(k-1)-4} z_{23}^{2 q}$ in $\left|T_{k-1}\right|$. Now, $a^{q} b \sim z_{34}^{2 q} z_{34}^{1}=z_{34}^{2 q} z_{34}^{2 q+2 k-4}$ and $a^{(k-1) q-1} b^{(k-1)} \sim$ $z_{34}^{2 q} z_{13}^{2 q+2 k-6} z_{23}^{2 q}=z_{34}^{2 q+2 k-5} z_{13}^{2 q+2 k-5} z_{23}^{2 q} \sim z_{23}^{2 q+2 k-5} z_{23}^{2 q}=z_{23}^{2 q+2 k-4} z_{23}^{2 q}$. Thus, $a^{k q-1} b^{k} \sim$ $\left(a^{q} b\right)\left(a^{(k-1) q-1} b^{(k-1)} \sim z_{34}^{2 q} z_{34}^{2 q+2 k-4} z_{23}^{2 q+2 k-4} z_{23}^{2 q}=z_{34}^{2 q} z_{13}^{2 q+2 k-4} z_{23}^{2 q}\right.$ in $\left|T_{k}\right|$. The claim now follows by induction.

Since $z_{13}^{2 q}=z_{13}^{2 q+2 k-4}$ in $T_{k}$, by the claim we get $a^{k q-1} b^{k} \sim 1$ in $\left|T_{k}\right|$. Thus, $\pi_{1}\left(\left|T_{k}\right|, v_{1}\right)=$ $\left\langle\alpha, \beta \mid \alpha^{k q-1} \beta^{k}, \alpha \beta \alpha^{-1} \beta^{-1}\right\rangle$ and hence $|T| \cup\left|T_{k}\right|=L(k q-1, k) \cong L(k q-1, q)$. Therefore, $\mathcal{M}_{k, q}$ is a crystallization of $L(k q-1, q)$.

\subsection{A $4(k+q)$-vertex crystallization of $L(k q+1, q)$}

Let $q \geqslant 4$. For each $k \geqslant 1$, we construct a $4(k+q)$-vertex 4-colored simple graph $\mathcal{N}_{k, q}=\left(\Gamma^{k}, \gamma^{k}\right)$ with the color set $\{1,2,3,4\}$ inductively which yields the presentation $\left\langle x, y \mid x^{q} y^{-1}, x y^{k}\right\rangle$. For this, we want $g_{12}^{k}=g_{34}^{k}=3$. Then, without loss, $g_{13}^{k}=g_{24}^{k}=k+q-1$ and $g_{14}^{k}=g_{23}^{k}=k+q$, where $g_{i j}^{k}$ is the number of components of $\Gamma_{i j}^{k}$ for $i \neq j$. These imply, $\Gamma_{14}^{k}$ and $\Gamma_{23}^{k}$ must be union of 4-cycles and $\Gamma_{13}^{k}$ (resp., $\Gamma_{24}^{k}$ ) has two 6-cycles and $(k+q-4)$ 4-cycles. Then, by Proposition $13, \mathcal{N}_{k, q}$ would be a crystallization of some connected closed 3-manifold $M_{k}$. 
$\boldsymbol{k}=\mathbf{1}$ case: The crystallization $\mathcal{N}_{1, q}$ is given in Fig. 8. Then, the components of $\Gamma_{12}^{1}$ are $G_{1}=C_{2 q}\left(x^{1}, \ldots, x^{2 q}\right), G_{2}=C_{4}\left(y^{1}, \ldots, y^{4}\right), G_{3}=C_{2 q}\left(z^{1}, \ldots, z^{2 q}\right)$ and the components of $\Gamma_{34}^{1}$ are $H_{1}=C_{2 q}\left(y^{3}, x^{2}, z^{2}, x^{4}, \ldots, z^{2 q-2}, x^{2 q}\right), H_{2}=C_{4}\left(z^{2 q-1}, x^{1}, z^{1}, y^{1}\right)$, $H_{3}=C_{2 q}\left(x^{3}, y^{2}, z^{2 q}, y^{4}, x^{2 q-1}, z^{2 q-3}, x^{2 q-3}, \ldots, z^{4}, x^{4}, z^{3}\right)$. Let $x, y$ be the generators corresponding to $G_{1}$ and $G_{2}$ respectively. To construct $\tilde{r}_{1}$ (resp., $\tilde{r}_{2}$ ) as in Eq. (2.6), choose $(i, j)=(4,3)$ and $v_{1}=y^{3}$ (resp., $\left.v_{1}=z^{2 q-1}\right)$. Then $H_{1}$ and $H_{2}$ represent the relations $x^{q} y^{-1}$ and $x y$ respectively. Therefore, by Proposition $14, \pi\left(M_{1}, *\right) \cong\left\langle x, y \mid x^{q} y^{-1}, x y\right\rangle \cong$ $\mathbb{Z}_{q+1}$.

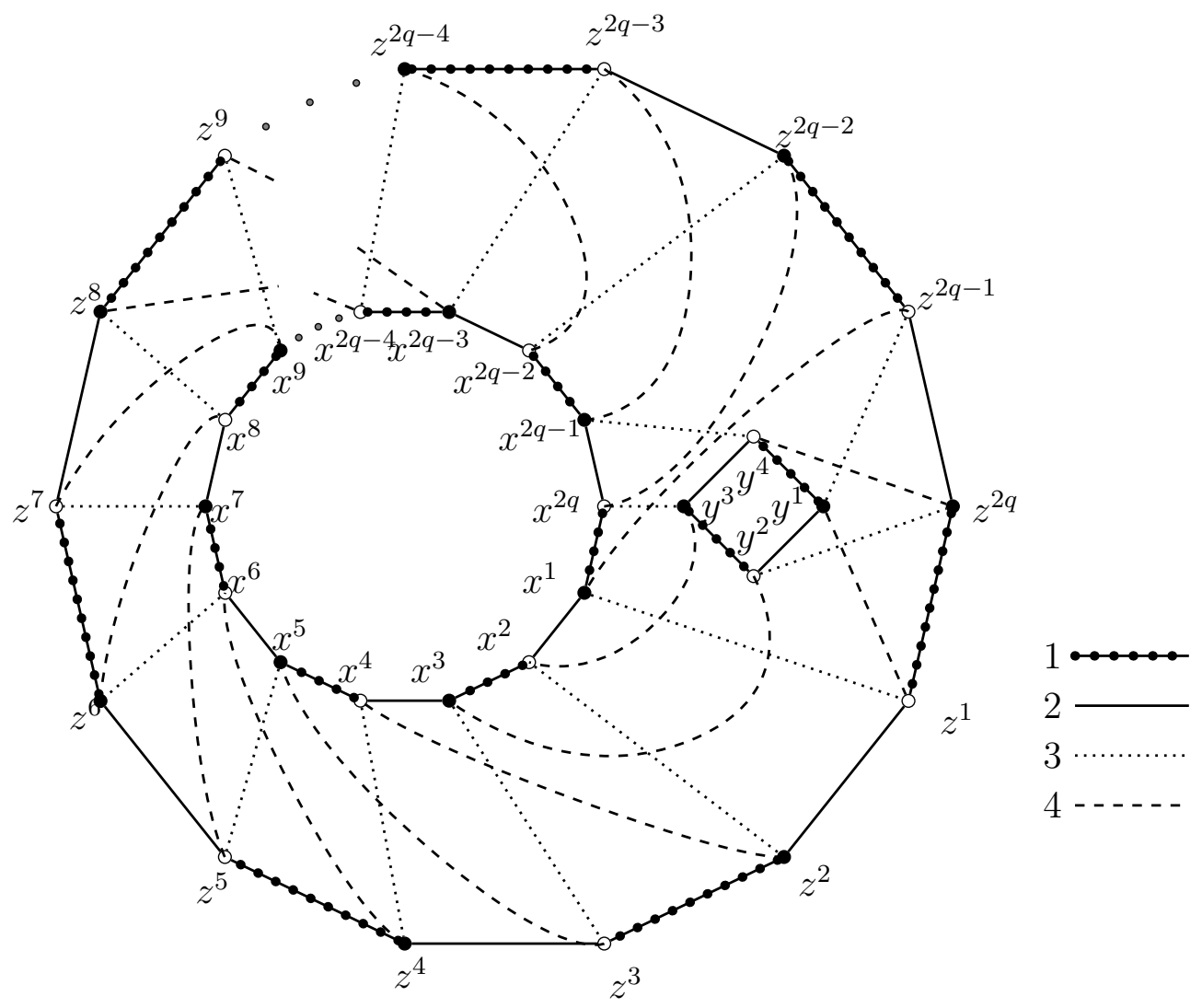

Figure 8: Crystallization $\mathcal{N}_{1, q}$ of $L(q+1, q)$

Let $T$ and $T_{1}$ be the 3 -dimensional simplicial cell complexes represented by the color graphs $\left.\Gamma^{1}\right|_{\left\{x^{5}, x^{4}, x^{3}, z^{3}\right\}}$ and $\left.\Gamma^{1}\right|_{V\left(\Gamma^{1}\right) \backslash\left\{x^{5}, x^{4}, x^{3}, z^{3}\right\}}$ respectively. Then $|T|$ and $\left|T_{1}\right|$ are solid tori and the facets (2-cells) of $T \cap T_{1}$ are $x_{2}^{5}, x_{3}^{5}, x_{3}^{4}, x_{4}^{4}, x_{1}^{3}, x_{4}^{3}, z_{1}^{3}, z_{2}^{3}$. Thus, $\left|T \cap T_{1}\right|$ is a torus (see Fig. 9 (b)) with $\pi_{1}\left(\left|T \cap T_{1}\right|, v_{1}\right)=\left\langle\alpha=[a], \beta=[b] \mid \alpha \beta \alpha^{-1} \beta^{-1}\right\rangle$, where $a=x_{34}^{4} x_{34}^{3}$ and $b=x_{34}^{3} x_{13}^{3} x_{23}^{4}$. Then $b=x_{34}^{3} x_{13}^{3} x_{23}^{3}=\partial\left(x_{3}^{1}\right) \sim 1$ in $|T|$. Therefore, $\pi_{1}\left(|T|, v_{1}\right)=\langle\alpha, \beta \mid \beta\rangle$.

Since $\alpha \beta=\beta \alpha$ in $\left|T \cap T_{1}\right|$, it follows that $a b \sim b a$ in $\left|T_{1}\right|$. Now, $a b=\left(x_{34}^{4} x_{34}^{3}\right)\left(x_{34}^{3} x_{13}^{3} x_{23}^{3}\right)$ $\sim x_{34}^{4} x_{13}^{3} x_{23}^{3}=z_{34}^{2} z_{13}^{2} x_{23}^{3} \sim z_{23}^{2} x_{23}^{3}=z_{23}^{1} x_{23}^{3}$. Thus, $a^{2} b \sim a b a \sim\left(z_{23}^{1} x_{23}^{3}\right)\left(x_{34}^{4} x_{34}^{3}\right)=$ $z_{23}^{1} x_{23}^{4} x_{34}^{4} x_{34}^{3} \sim z_{23}^{1} x_{13}^{4} x_{34}^{3}=z_{23}^{1} x_{13}^{5} x_{34}^{5} \sim z_{23}^{1} x_{23}^{5}=z_{23}^{1} x_{23}^{6}$. Therefore, $a^{3} b \sim a b a^{2} \sim$ $z_{23}^{1} x_{23}^{6} x_{34}^{4} x_{34}^{3}=z_{23}^{1} x_{23}^{6} x_{34}^{6} x_{34}^{3} \sim z_{23}^{1} x_{13}^{6} x_{34}^{3}=z_{23}^{1} x_{13}^{7} x_{34}^{7} \sim z_{23}^{1} x_{23}^{7}=z_{23}^{1} x_{23}^{8}$. Similarly we get, $a^{q-2} b \sim z_{23}^{1} x_{23}^{2 q-2}$. Thus, $a^{q-1} b \sim a^{q-2} b a \sim z_{23}^{1} x_{23}^{2 q-2} x_{34}^{4} x_{34}^{3}=z_{23}^{1} x_{23}^{2 q-2} x_{34}^{2 q-2} x_{34}^{3} \sim$ $z_{23}^{1} x_{13}^{2 q-2} x_{34}^{3}=z_{23}^{1} x_{13}^{2 q-1} x_{34}^{2 q-1} \sim z_{23}^{1} x_{23}^{2 q-1}=z_{23}^{1} x_{23}^{2 q}$. Therefore, $a^{q} b \sim z_{23}^{1} x_{23}^{2 q} x_{34}^{4} x_{34}^{3}=$ 
$z_{23}^{1} x_{23}^{2 q} x_{34}^{2 q} x_{34}^{3} \sim z_{23}^{1} x_{13}^{2 q} x_{34}^{3}=z_{23}^{1} y_{13}^{2} y_{34}^{2} \sim z_{23}^{1} y_{23}^{2} \sim z_{34}^{1} z_{13}^{1} y_{23}^{2}=z_{34}^{1} y_{13}^{2} y_{23}^{2} \sim z_{34}^{1} y_{34}^{2}=z_{34}^{1} z_{34}^{2 q}$. Again, $a=x_{34}^{4} x_{34}^{3}=y_{34}^{3} y_{34}^{2} \sim y_{24}^{3} y_{14}^{3} y_{34}^{2}=y_{24}^{3} y_{14}^{2} y_{34}^{2} \sim y_{24}^{3} y_{24}^{2}=y_{24}^{3} z_{24}^{1}$. Therefore, $a^{q+1} b \sim a a^{q} b \sim y_{24}^{3} z_{24}^{1} z_{34}^{1} z_{34}^{2 q} \sim z_{24}^{2 q} z_{14}^{1} z_{34}^{2 q}=z_{24}^{2 q} z_{14}^{2 q} z_{34}^{2 q}=\partial\left(z_{4}^{2 q}\right) \sim 1$ in $\left|T_{1}\right|$. Thus $\pi_{1}\left(\left|T_{1}\right|, v_{1}\right)=\left\langle\alpha, \beta \mid \alpha^{q+1} \beta, \alpha \beta \alpha^{-1} \beta^{-1}\right\rangle$. This implies that $|T| \cup\left|T_{1}\right|=L(q+1,1)$. Therefore, $\mathcal{N}_{1, q}$ is a crystallization of $L(q+1,1) \cong L(q+1, q)$.

$\boldsymbol{k}=\mathbf{2}$ case: Here $z_{14}^{1} \neq z_{14}^{2 q}$. Let

$$
\begin{aligned}
\Gamma^{2}= & \left(V\left(\Gamma^{1}\right) \cup\left\{y^{5}, y^{6}, z^{2 q+1}, z^{2 q+2}\right\}, E\left(\Gamma^{1}\right) \backslash\left\{y^{2} z^{2 q}, y^{1} z^{2 q-1}, y^{1} y^{4}, z^{1} z^{2 q}\right\} \cup\left\{y^{1} y^{6}, y^{5} y^{6},\right.\right. \\
& \left.\left.y^{4} y^{5}, z^{1} z^{2 q+2}, z^{2 q+1} z^{2 q+2}, z^{2 q} z^{2 q+1}, y^{5} z^{2 q+1}, y^{6} z^{2 q+2}, y^{2} z^{2 q+2}, y^{1} z^{2 q+1}, y^{6} z^{2 q}, y^{5} z^{2 q-1}\right\}\right) .
\end{aligned}
$$

To construct $\mathcal{N}_{2, q}$, consider the following coloring $\gamma^{2}$ on the edges of $\Gamma^{2}$ : same colors on the old edges as in $\mathcal{N}_{1, q}$, color 1 on the edges $y^{1} y^{6}, y^{4} y^{5}, z^{1} z^{2 q+2}, z^{2 q} z^{2 q+1}$ color 2 on the edges $y^{5} y^{6}, z^{2 q+1} z^{2 q+2}$, color 3 on the edges $y^{2} z^{2 q+2}, y^{1} z^{2 q+1}, y^{6} z^{2 q}, y^{5} z^{2 q-1}$ and color 4 on the edges $y^{5} z^{2 q+1}, y^{6} z^{2 q+2}$ (see Fig. $9(a)$ ). Let $T$ be as in the case $k=2$ and $T_{2}$ be the cell complex represented by the colored graph $\left.\Gamma^{2}\right|_{V\left(\Gamma^{2}\right) \backslash\left\{x^{5}, x^{4}, x^{3}, z^{3}\right\}}$.
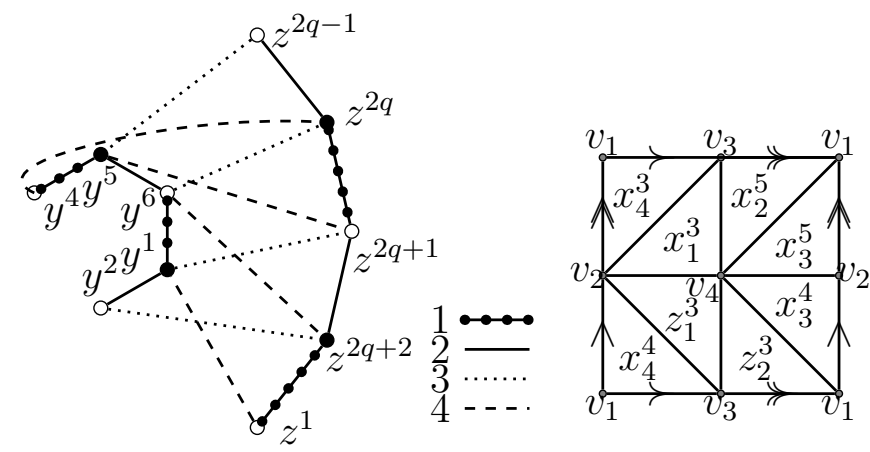

Figure 9: (a) Crystallization $\mathcal{N}_{2, q}$ of $L(2 q+1, q)$

(b) $|T| \cap\left|T_{k}\right|$

Then, $a^{q+1} b \sim z_{24}^{2 q} z_{14}^{1} z_{34}^{2 q}=z_{24}^{2 q} z_{14}^{2 q+2} z_{34}^{2 q+2} \sim z_{24}^{2 q} z_{24}^{2 q+2}=z_{24}^{2 q} z_{24}^{2 q+1}$. This implies, $a^{2 q+1} b^{2}$ $\sim a^{q+1} b a^{q} b \sim z_{24}^{2 q} z_{24}^{2 q+1} z_{34}^{1} z_{34}^{2 q}=z_{24}^{2 q} z_{24}^{2 q+1} z_{34}^{2 q+1} z_{34}^{2 q} \sim z_{24}^{2 q} z_{14}^{2 q+1} z_{34}^{2 q}=z_{24}^{2 q} z_{14}^{2 q} z_{34}^{2 q}=\partial\left(z_{4}^{2 q}\right) \sim 1$ in $\left|T_{2}\right|$. Thus, $\pi_{1}\left(\left|T_{2}\right|, v_{1}\right)=\left\langle\alpha, \beta \mid \alpha^{2 q+1} \beta^{2}, \alpha \beta \alpha^{-1} \beta^{-1}\right\rangle$ and hence $|T| \cup\left|T_{2}\right|=L(2 q+1,2)$. Therefore, $\mathcal{N}_{2, q}$ is a crystallization of $L(2 q+1,2) \cong L(2 q+1, q)$.

$\boldsymbol{k} \geqslant 3$ case: Let

$$
\begin{gathered}
\Gamma^{k}=\left(V\left(\Gamma^{k-1}\right) \cup\left\{y^{2 k+1}, y^{2 k+2}, z^{2 q+2 k-3}, z^{2 q+2 k-2}\right\}, E\left(\Gamma^{k-1}\right) \backslash\left\{y^{2 k} z^{2 q}, y^{2 k-1} z^{2 q-1}, y^{2 k-1} y^{4},\right.\right. \\
\left.z^{2 q+2 k-5} z^{2 q}\right\} \cup\left\{y^{2 k-1} y^{2 k+2}, y^{2 k+1} y^{2 k+2}, z^{2 q+2 k-5} z^{2 q+2 k-2}, z^{2 q+2 k-3} z^{2 q+2 k-2}, z^{2 q} z^{2 q+2 k-3},\right. \\
\left.\left.y^{2 k+1} z^{2 q+2 k-3}, y^{2 k+2} z^{2 q+2 k-2}, y^{2 k} z^{2 q+2 k-2}, y^{2 k-1} z^{2 q+2 k-3}, y^{2 k+2} z^{2 q}, y^{2 k+1} z^{2 q-1}, y^{4} y^{2 k+1}\right\}\right) .
\end{gathered}
$$

To construct $\mathcal{N}_{k, q}$, consider the following coloring $\gamma^{k}$ on the edges of $\Gamma^{k}$ : same colors on the old edges as in $\mathcal{N}_{k-1, q}$, color 1 on the edges $y^{2 k-1} y^{2 k+2}, y^{4} y^{2 k+1}, z^{2 q+2 k-5} z^{2 q+2 k-2}$, $z^{2 q} z^{2 q+2 k-3}$, color 2 on the edges $y^{2 k+1} y^{2 k+2}, z^{2 q+2 k-3} z^{2 q+2 k-2}$, color 3 on the edges $y^{2 k} z^{2 q+2 k-2}, y^{2 k-1} z^{2 q+2 k-3}, y^{2 k+2} z^{2 q}, y^{2 k+1} z^{2 q-1}$ and color 4 on the edges $y^{2 k+1} z^{2 q+2 k-3}$, $y^{2 k+2} z^{2 q+2 k-2}$. Let $T$ be as in the case $k=1$ and $T_{k}$ be the cell complex represented by the colored graph $\left.\Gamma^{k}\right|_{V\left(\Gamma^{k}\right) \backslash\left\{x^{5}, x^{4}, x^{3}, z^{3}\right\}}$. 
Claim. $a^{k q+1} b^{k} \sim z_{24}^{2 q} z_{14}^{2 q+2 k-3} z_{34}^{2 q}$ in $\left|T_{k}\right|$.

We prove the claim by induction. It is true for $k=2$. Assume that $a^{(k-1) q+1} b^{k-1} \sim$ $z_{24}^{2 q} z_{14}^{2 q+2(k-1)-3} z_{34}^{2 q}$ in $\left|T_{k-1}\right|$. Now $a^{q} b \sim z_{34}^{2 q+2 k-3} z_{34}^{2 q}$ and $a^{q(k-1)+1} b^{(k-1)} \sim z_{24}^{2 q} z_{24}^{2 q+2 k-4}=$ $z_{24}^{2 q} z_{24}^{2 q+2 k-3}$. So, $a^{q k+1} b^{k} \sim\left(a^{q(k-1)+1} b^{(k-1)}\left(a^{q} b\right) \sim z_{24}^{2 q} z_{24}^{2 q+2 k-3} z_{34}^{2 q+2 k-3} z_{34}^{2 q}=z_{24}^{2 q} z_{14}^{2 q+2 k-3}\right.$ in $\left|T_{k}\right|$. The claim now follows by induction.

Since $z_{14}^{2 q}=z_{14}^{2 q+2 k-3}$ in $T_{k}$, by the claim we get $a^{k q+1} b^{k} \sim 1$ in $\left|T_{k}\right|$. Thus, $\pi_{1}\left(\left|T_{k}\right|, v_{1}\right)=$ $\left\langle\alpha, \beta \mid \alpha^{k q+1} \beta^{k}, \alpha \beta \alpha^{-1} \beta^{-1}\right\rangle$ and hence $|T| \cup\left|T_{k}\right|=L(k q+1, k) \cong L(k q+1, q)$. Therefore, $\mathcal{N}_{k, q}$ is a crystallization of $L(k q+1, q)$.

A few days after we posted the first version of this article (arXiv:1308.6137) in the arXiv, Casali and Cristofori posted an article on complexity of lens spaces [4] in the arXiv (arXiv:1309.5728). In that paper, the authors constructed crystallizations of $L(p, q)$ with $4 S(p, q)$ vertices, where $S(p, q)$ denotes the sum of all partial quotients in the expansion of $q / p$ as a regular continued fraction. In particular, they have constructed $L(k q-1, q)$ with $4(k+q-1)$ vertices for $k, q \geqslant 2$ and $L(k q+1, q)$ with $4(k+q)$ vertices for $k, q \geqslant 1$. Their constructions are different from ours.

Remark 25. From the enumeration of crystallizations of prime 3-manifolds with at most 30 vertices (see $[3,12]$ ), we know that $\Psi(L(9,4))=24$ and $\Psi(L(13,4))=28$. From our constructions in Subsections 5.1 and 5.2, we know $\mathcal{M}_{2,5}$ and $\mathcal{N}_{2,4}$ are 24-vertex crystallizations of $L(9,4)$. The induced subgraphs of $\mathcal{M}_{2,5}$ on 2-colored edges are of the form $2 C_{10} \sqcup C_{4}, 2 C_{6} \sqcup 3 C_{4}$ or $6 C_{4}$ and such subgraphs of $\mathcal{N}_{2,4}$ are of the form $C_{10} \sqcup C_{8} \sqcup C_{6}$, $2 C_{6} \sqcup 3 C_{4}$ or $6 C_{4}$. So, $\mathcal{M}_{2,5}$ and $\mathcal{N}_{2,4}$ are non-isomorphic. Thus, $L(9,4)$ has more than one (non-isomorphic) crystallizations with minimum number of vertices. The constructions in [4] give a 28-vertex of crystallization of $L(13,4)$ with $\left\{g_{12}, g_{13}, g_{14}\right\}=\{4,5,7\}$. Observe that $\mathcal{N}_{3,4}$ is also a 28 -vertex of crystallization of $L(13,4)$ with $\left\{g_{12}, g_{13}, g_{14}\right\}=\{3,6,7\}$. Thus, these two crystallizations of $L(13,4)$ are non-isomorphic. So, the minimal crystallization $\mathcal{N}_{3,4}$ of $L(13,4)$ is not unique. Also, from the list of crystallizations in [12], we know that there are several 3-manifolds having more than one crystallizations with minimum number of vertices.

\section{Proofs of Theorems 6, 8 and Corollary 7}

Proof of Theorem 6. Let $\mathcal{M}_{2,3}$ be as in Subsection 5.1. Then, $\mathcal{M}_{2,3}$ is a 16 -vertex crystallization of $L(5,3)=L(5,2)$. Part (i) now follows from Lemmas $15,19, \ldots, 23$.

If $f_{3}(X)<8$ then, by Theorem $4, \psi(M)<8$ and hence $\psi(M)=2$. Therefore $\pi(M, *)=\{0\}$ and hence, by Perelman's theorem (Poincaré conjecture), $M=S^{3}$. Part (ii) now follows from Lemma 19.

Proof of Corollary 7. From the proof of Lemma 22, $m\left(Q_{8}\right)=2$. Therefore, if $X$ is a pseudotriangulation of $S^{3} / Q_{8}$ then, by Corollary 5 and Lemma $3, h_{2}(X) \geqslant \psi\left(S^{3} / Q_{8}\right)-2=$ $18-2>12=6 m\left(S^{3} / Q_{8}\right)$.

Again, if $X$ is a pseudotriangulation of $S^{1} \times S^{1} \times S^{1}$ then, by Corollary 5 and Lemma $3, h_{2}(X) \geqslant \psi\left(S^{1} \times S^{1} \times S^{1}\right)-2=24-2>6 \times 3=6 m\left(S^{1} \times S^{1} \times S^{1}\right)$. 
For $p, q$ relatively prime and $p \geqslant 3$, let $X$ be a pseudotriangulation of $L(p, q)$. Then, by Theorem 6 (ii) and Corollary $5, h_{2}(X) \geqslant \psi(L(p, q))-2>8-2=6 \times 1=6 m(L(p, q))$ for $p \geqslant 3$. This completes the proof.

Proof of Theorem 8. Let $\mathcal{K}_{p, q}$ be as in Remark 24. Then $\mathcal{K}_{3,1}$ is a 12 -vertex crystallization of $L(3,2)$. Part (a) now follows by the constructions in Subsection 5.1.

Again, $\mathcal{K}_{q+1, q}$ is a $4(q+1)$-vertex crystallization of $L(q+1, q)$ for $1 \leqslant q \leqslant 3$. Part (b) now follows by the constructions in Subsection 5.2.

\section{Acknowledgement}

This work is supported in part by UGC Centre for Advanced Studies. The first author thanks CSIR, India for SPM Fellowship. The authors thank M. R. Casali and P. Cristofori for pointing out an error in an earlier version of this paper. The authors also thank the anonymous referee for many useful comments.

\section{References}

[1] A. Björner, Posets, regular CW complexes and Bruhat order, European J. Combin. 5 (1984), 7-16.

[2] J. A. Bondy and U. S. R. Murty, Graph Theory, Springer, New York, 2008.

[3] M. R. Casali and P. Cristofori, A catalogue of orientable 3-manifolds triangulated by 30 coloured tetrahedra, J. Knot Theory Ramification 17 (2008), 1-23.

[4] M. R. Casali and P. Cristofori, A note about complexity of lens spaces, arXiv: 1309.5728, 2013.

[5] A. Cavicchioli, L. Grasselli and M. Pezzana, Su di una decomposizione normale per le $n$-varietà chiuse, Boll. Un. Mat. Ital. 17-B (1980), 1146-1165.

[6] D. B. A. Epstein, Finite presentations of groups and 3-manifolds, Quart. J. Math. Oxford 12 (1961), 205-212.

[7] M. Ferri and C. Gagliardi, Crystalization moves, Pacific J. Math. 100 (1982), 85-104.

[8] M. Ferri, C. Gagliardi and L. Grasselli, A graph-theoretic representation of PLmanifolds - a survey on crystallizations, Acquationes Math. 31 (1986), 121-141.

[9] C. Gagliardi, A combinatorial characterization of 3-manifold crystallizations, Boll. Un. Mat. Ital. 16-A (1979), 441-449.

[10] C. Gagliardi, How to deduce the fundamental group of a closed $n$-manifold from a contracted triangulation, J. Combin. Inform. System Sci. 4 (1979), 237-252.

[11] S. Klee, The fundamental group of balanced simplicial complexes and posets, Electron. J Combin. 16 (2) (2009), \#R7.

[12] S. Lins, Gems, computers and attractors for 3-manifolds, Series on Knots and Everything 5, World Scientific, River Edge, NJ, 1995. 
[13] S. Murai, Face vectors of simplicial cell decompositions of manifolds, Israel J. Math. 195 (2013), 187-213.

[14] G. Perelman, Finite extinction time for the solutions to the Ricci flow on certain three-manifolds, arXiv:math/0307245, 2003.

[15] M. Pezzana, Sulla struttura topologica delle varietà compatte, Atti Sem. Mat. Fis. Univ. Modena 23 (1974), 269-277.

[16] E. Swartz, The average dual surface of a cohomology class and minimal simplicial decompositions of infinitely many lens spaces, arXiv:1310.1991, 2013. 